\title{
A DETAILED PROOF OF A THEOREM OF AUBIN
}

\author{
FARID MADANI
}

ABSTRACT. In this note we give a detailed proof of a theorem of Aubin, namely [1 Théorème 5].

\section{INTRODUCTION}

The goal of this note is to give a detailed proof of the following theorem of Aubin.

Theorem 1 (Aubin [1). Let $(M, g)$ be a compact Riemannian manifold of dimension $n$. Assume that, in a geodesic normal coordinate chart around a point $x \in M$, $\operatorname{det}(g)=1+O\left(r^{N}\right)$, with $N$ sufficiently large, where $r=d(x, \cdot)$. Let $\omega$ denote $\omega:=\inf \left\{k \in \mathbb{N}:\left|\nabla^{k} W_{\text {eyl }}(x)\right| \neq 0\right\}$. If $\left|\nabla^{\omega} \operatorname{scal}(x)\right|=0$, then $\Delta^{\omega+1} \operatorname{scal}(x)<0$. In particular, $\int_{\partial B_{r}(x)}$ scal $\mathrm{d} \sigma<0$, for $r$ sufficiently small.

For the particular values of $\omega \in\{1,2\}$, Theorem 1 is proven by Hebey and Vaugon [6. The case $\omega=3$ was studied by L. Zhang, as mentioned in [1.

Theorem 1 is fundamental in questions related to the compactness of the Yamabe equation solutions and the equivariant Yamabe problem. For example, in [8, 9, the author used Theorem 1 to prove the validity of the Hebey-Vaugon conjecture, when $\omega \leq \frac{n-6}{2}$ (more details are given in Section 2).

Aubin used Theorem 1 in 1, 2, 3 in his study of the compactness of the set of solutions to the Yamabe equation. He claimed the compactness of this set except for the round sphere. This claim is in a contradiction with the counter examples constructed by Brendle 4 and by Brendle and Marques [5. Many mathematicians had serious concerns about the correctness of 1, although it remained unclear which parts of [1 are correct. For example, the proof of [1, Théorème 5] is only sketched and not given in all details, and thus it is difficult for a reader to check whether the result of this theorem holds or not. The aim of this note is to provide a rigorous proof of [1, Théorème 5].

In our proof of Theorem 1, we did not follow exactly the strategy proposed by Aubin in [1. However, there are certain common points. For example, the formula in Corollary 7 coincides with the last formula on page 279 in [1.

In order to facilitate the reading, we gathered all notation in Notation Index A.2

\section{The equivariant Yamabe Problem}

In this section, we present an application of Theorem 1 . Let $(M, g)$ be a compact Riemannian manifold of dimension $n \geq 3$ and $G$ be a subgroup of the isometry group $\operatorname{Isom}(M, g)$. The equivariant Yamabe problem consists in finding a $G$-invariant metric in the conformal class of $g$, with constant scalar curvature. Hebey and Vaugon proved that a sufficient condition to solve this problem is to prove that the following conjecture holds.

Key words and phrases. scalar curvature; tensor symmetrizations; 
Hebey-Vaugon conjecture. If $(M, g)$ is not conformal to the round sphere $S^{n}$ or if the action of $G$ has no fixed point, then the following strict inequality holds

$$
\inf _{g^{\prime} \in[g]^{G}} \frac{\int_{M} \mathrm{scal}_{g^{\prime}} \mathrm{d} v_{g^{\prime}}}{\left(\int_{M} \mathrm{~d} v_{g^{\prime}}\right)^{\frac{n-2}{n}}}<n(n-1) \omega_{n}^{2 / n}\left(\inf _{x \in M} \operatorname{card}(G \cdot x)\right)^{2 / n},
$$

where $\omega_{n}$ is the volume of round sphere $S^{n}$.

For $\omega \leq 2$ Hebey and Vaugon [6] proved Theorem 1 and they used this result to prove the above conjecture. They also proved it for $\omega>\frac{n-6}{2}$, assuming the positive mass theorem.

In [8, 9, the author used Theorem 1 to prove the validity of the Hebey-Vaugon conjecture for $\omega \leq \frac{n-6}{2}$, by constructing a local $G$-invariant positive test function, which involves the scalar curvature, where the negative sign of $\int_{\partial B_{r}(x)}$ scal $\mathrm{d} \sigma$ plays an important role. For more details, see [6], 8, 9] and the references therein.

\section{The Hebey-Vaugon formulas}

Let us first mention some notation and convention used throughout this note. Our convention for the components of the Riemann and Ricci curvature tensors are: in a local normal coordinate chart,

$$
R_{j k \ell}^{q} X^{j}=\left(\nabla_{k} \nabla_{\ell} X\right)^{q}-\left(\nabla_{\ell} \nabla_{k} X\right)^{q}, \quad\left(\nabla_{i j} \alpha\right)_{k}=\left(\nabla_{j i} \alpha\right)_{k}-R_{k i j}^{\ell} \alpha_{\ell},
$$

for any vector field $X$ and 1-form $\alpha$. Moreover, we set $R_{i j k \ell}=g_{i q} R_{j k \ell}^{q}, \operatorname{Ric}_{i j}=R_{i \ell j}^{\ell}$. For any $k$-covariant tensor $T$, we have:

$$
\nabla_{q} T_{p_{1} \ldots p_{k}}:=\left(\nabla_{q} T\right)_{p_{1} \ldots p_{k}}, \quad \Delta T_{p_{1} \ldots p_{k}}:=\left(g^{p q} \nabla_{p q} T\right)_{p_{1} \ldots p_{k}}
$$

We define the symmetrization of the tensor $T$ by

$$
\operatorname{Sym} T_{I}:=\operatorname{Sym}_{p_{1}, \ldots, p_{k}} T_{p_{1} \ldots p_{k}}:=\sum_{\sigma \in \mathfrak{S}(k)} T_{p_{\sigma(1)} \ldots p_{\sigma(k)}}
$$

where $\mathfrak{S}(k)$ is the symmetric group on the finite set $\{1, \ldots, k\}$ and $I=\left(p_{1}, \ldots, p_{k}\right)$ is a multi-index $k$-tuple. The two-by-two contraction of $\operatorname{Sym} T_{I}$, denoted by $\operatorname{Tr} \operatorname{Sym} T_{I}$, is defined by

$$
\operatorname{Tr} \operatorname{Sym}_{I}:=\left\{\begin{array}{l}
g^{p_{1} p_{2}} \ldots g^{p_{k-1} p_{k}} \operatorname{Sym} T_{p_{1} \ldots p_{k}}, \text { if } k \text { is even } \\
g^{p_{1} p_{2}} \ldots g^{p_{k-2} p_{k-1}} \operatorname{Sym} T_{p_{1} \ldots p_{k}}, \text { if } k \text { is odd }
\end{array}\right.
$$

Note that when $k$ is odd, the two-by-two contraction of SymT is a 1-covariant tensor. We always use the Einstein summation convention, i.e. each time an index occurs twice we sum over it.

Let $(M, g)$ be a compact Riemannian manifold of dimension $n$. We fix $x \in M$ and define: $\omega=\inf \left\{k \in \mathbb{N}:\left|\nabla^{k} W e y l_{g}(x)\right| \neq 0\right\}$. Lee and Parker [7] proved that in each conformal class $[g]$, there exists a metric $g^{\prime}$ which satisfies, in a geodesic normal chart, $\operatorname{det}\left(g^{\prime}\right)=1+O\left(r^{N}\right)$, with $N$ sufficiently large, where $r=d(x, \cdot)$. This result was extended by Hebey and Vaugon [6] in the equivariant setting. If $G$ is a subgroup of the isometry group $\operatorname{Isom}(M, g)$ and $[g]^{G}$ denotes the $G$-invariant conformal class of $g$, then they proved

Lemma 2. In each class $[g]^{G}$, there exists a metric $g^{\prime}$ which satisfies, in a geodesic normal chart, $\operatorname{det}\left(g^{\prime}\right)=1+O\left(r^{N}\right)$, with $N$ sufficiently large, where $r=d(x, \cdot)$. 
Moreover, in this chart, the Taylor expansion of $g^{\prime}$ is given by

$$
\begin{gathered}
g_{i j}^{\prime}(y)=\delta_{i j}+\sum_{\omega+4 \leq m \leq 2 \omega+6} \frac{2(m-3)}{(m-1) !} \nabla_{p_{3} \ldots p_{m-2}} R_{i p_{1} p_{2} j}^{\prime}(x) x^{p_{1}} \ldots x^{p_{m-2}}+ \\
K(\omega) \sum_{k=1}^{n} \nabla_{p_{3} \ldots p_{\omega+2}} R_{i p_{1} p_{2} k}^{\prime}(x) \nabla_{p_{\omega+5} \ldots p_{2 \omega+4}} R_{j p_{\omega+3} p_{\omega+4} k}^{\prime}(x) x^{p_{1}} \ldots x^{p_{2 \omega+4}}+O\left(r^{2 \omega+5}\right),
\end{gathered}
$$

where $K(\omega)=\frac{(3 \omega+8)(\omega+1)^{2}}{(2 \omega+5)[(\omega+3) !]^{2}}$.

Using the above Taylor expansion of the metric and writing the metric as exponential of some symmetric matrix, Hebey and Vaugon proved the following formulas.

Theorem 3 (Hebey-Vaugon [6]). Let $(M, g)$ be a compact Riemannian manifold of dimension $n$. Assume that $\operatorname{det}(g)=1+O\left(r^{N}\right)$, with $N$ sufficiently large, in a geodesic normal coordinate chart around $x$. Then the following statements hold

1. For any nonnegative integer $k \leq \omega-1,\left|\nabla^{k} \operatorname{Riem}(x)\right|=0$.

2. For any nonnegative integer $k \leq 2 \omega+1$, the $k$-th covariant derivatives of the Riemann, Ricci and scalar curvatures of $g^{\prime}$ coincide with the usual partial derivatives and thus they commute. Namely, for any multi-index $\beta \in\{1, \ldots, n\}^{k}$,

$$
\begin{aligned}
& \nabla_{\beta} \operatorname{Riem}(x)=\partial_{\beta} \operatorname{Riem}(x), \nabla_{\beta} \operatorname{Ric}(x)=\partial_{\beta} \operatorname{Ric}(x), \nabla_{\beta} \operatorname{scal}_{g}(x)=\partial_{\beta} \operatorname{scal}_{g}(x) . \\
& \text { 3. For any } \omega+2 \leq m \leq 2 \omega+3, \underset{p_{1} \ldots p_{m}}{\operatorname{Sym}} \nabla_{p_{3} \ldots p_{m}} \operatorname{Ric}_{p_{1} p_{2}}(x)=0 \text { and } \\
& \underset{p_{1} \ldots p_{2 \omega+4}}{\operatorname{Sym}}\left\{\nabla_{p_{3} \ldots p_{2 \omega+4}} \operatorname{Ric}_{p_{1} p_{2}}(x)\right. \\
& \left.\quad+C(\omega) \nabla_{p_{3} \ldots p_{\omega+2}} R_{i p_{1} p_{2} j}(x) \cdot \nabla_{p_{\omega+5} \ldots p_{2 \omega+4}} R_{i p_{\omega+3} p_{\omega+4} j}(x)\right\}=0,
\end{aligned}
$$

where $C(\omega)=(\omega+1)^{2}(\omega+2)^{2}(2 \omega+2) ![(\omega+3) !]^{-2}$.

Assume that $g$ satisfies the assumptions of Theorem 3. It follows from the theorem that $\Delta^{\ell}$ scal $(x)=0$ and $\left|\nabla \Delta^{\ell} \operatorname{scal}(x)\right|=0$, for any nonnegative integer $\ell \leq \omega$. Indeed, Tr $\underset{p_{1} \ldots p_{2 \ell}}{\operatorname{Sym}} \nabla_{p_{1} \ldots p_{2 \ell}} \operatorname{scal}(x)=(2 \ell) ! \Delta^{\ell} \operatorname{scal}(x)$ and $\underset{p_{1} \ldots p_{2 \ell+3}}{\operatorname{Sym}} \nabla_{p_{3} \ldots p_{2 \ell+3}} \operatorname{Ric}_{p_{1} p_{2}}(x)=$ Sym $\left\{2 \nabla_{p_{2} \ldots p_{2 \ell+2}} \operatorname{Ric}_{p_{1} p_{2 \ell+3}}(x)+(2 \ell+1) \nabla_{p_{3} \ldots p_{2 \ell+3}} \operatorname{Ric}_{p_{1} p_{2}}(x)\right\}$, since by Theorem 3 , the covariant derivatives of order at most $2 \omega+1$ of the Ricci curvature tensor and the scalar curvature commute. It follows that

$$
\begin{gathered}
2(2 \ell) !(\ell+1)^{2} \Delta^{\ell} \operatorname{scal}(x)=\operatorname{Tr} \underset{p_{1} \ldots p_{2 \ell+2}}{\operatorname{Sym}} \nabla_{p_{3} \ldots p_{2 \ell+2}} \operatorname{Ric}_{p_{1} p_{2}}(x)=0, \\
(2 \ell+2) !(\ell+2) \nabla_{p_{2 \ell+3}} \Delta^{\ell} \operatorname{scal}(x)=\operatorname{Tr} \underset{p_{1} \ldots p_{2 \ell+3}}{\operatorname{Sym}} \nabla_{p_{3} \ldots p_{2 \ell+3}} \operatorname{Ric}_{p_{1} p_{2}}(x)=0 .
\end{gathered}
$$

Moreover, if we assume that $\left|\nabla^{\omega} \operatorname{scal}(x)\right|=0$, then the $(2 \omega+2)$-covariant derivatives of the scalar curvature at $x$ commute (this fact will be proven at the end of the proof of Proposition 6). For $r=d(x, \cdot)$ sufficiently small, one can write the Taylor expansion of the scalar curvature in $B_{r}(x)$ and prove that there exists a positive constant $c(n, \omega)$ depending on $n$ and $\omega$, such that $\frac{1}{\operatorname{vol}\left(\partial B_{r}(x)\right)} \int_{\partial B_{r}(x)} \operatorname{scal} \mathrm{d} \sigma=$ $c(n, \omega) \sum_{\ell=0}^{\omega+1} \Delta^{\ell} \operatorname{scal}(x) r^{2 \ell}+O\left(r^{2 \omega+3}\right)$. We conclude that

$$
\frac{1}{\operatorname{vol}\left(\partial B_{r}(x)\right)} \int_{\partial B_{r}(x)} \operatorname{scal} \mathrm{d} \sigma=c(n, \omega) \Delta^{\omega+1} \operatorname{scal}(x) r^{2 \omega+2}+O\left(r^{2 \omega+3}\right) .
$$

Thus, $\int_{\partial B_{r}(x)} \operatorname{scal} \mathrm{d} \sigma$ and $\Delta^{\omega+1} \operatorname{scal}(x)$ have the same sign. 


\section{RiCCI SYMMETRIZATION}

From now on, $(M, g)$ denotes a compact Riemannian manifold of dimension $n$. We fix a point $x \in M$ and assume that $\operatorname{det}(g)=1+O\left(r^{N}\right)$, with $N$ sufficiently large, in a geodesic normal coordinate chart around $x$ (in particular, $g_{i j}(x)=\delta_{i j}$ ) and that all the covariant derivatives of order $\omega$ of the scalar curvature at $x$ vanish, i.e. $\left|\nabla^{\omega} \operatorname{scal}(x)\right|=0$. Since all the tensors are evaluated at $x$, we omit to mention $x$ for all tensors. For example, we write $\nabla_{I} \operatorname{Ric}_{i j}$ instead of $\nabla_{I} \operatorname{Ric}_{i j}(x)$.

We introduce the following notation:

$$
\begin{gathered}
\mathcal{R}_{\ell}=\left.\left|\nabla^{\omega-2 \ell} \Delta^{\ell} \operatorname{Riem}^{2}, \quad \mathcal{T}_{\ell}=\right| \nabla^{\omega-2 \ell} \Delta^{\ell} \operatorname{Ric}\right|^{2}, \quad 0 \leq \ell \leq \frac{\omega}{2}, \\
\mathcal{M}_{\ell}=\nabla_{K} \nabla_{a} \Delta^{\ell} \operatorname{Ric}_{b c} \cdot \nabla_{K} \nabla_{c} \Delta^{\ell} \operatorname{Ric}_{a b}, \quad 0 \leq \ell \leq \frac{\omega-1}{2}, \\
\mathcal{N}_{\ell}=\nabla_{K^{\prime}} \nabla_{c d} \Delta^{\ell} \operatorname{Ric}_{a b} \cdot \nabla_{K^{\prime}} \nabla_{a b} \Delta^{\ell} \operatorname{Ric}_{c d}, \quad 0 \leq \ell \leq \frac{\omega-2}{2},
\end{gathered}
$$

where we denote by $K$ and $K^{\prime}$ two multi-indices $(\omega-2 \ell-1)$ and $(\omega-2 \ell-2)$-tuples respectively, and the fixed point $x$ is omitted. By convention, $\mathcal{N}_{\frac{\omega}{2}}=\mathcal{M}_{\frac{\omega}{2}}=0$, if $\omega$ is even and $\mathcal{N}_{\frac{\omega-1}{2}}=0$, if $\omega$ is odd.

Lemma 4. For any $\ell \leq \frac{\omega}{2}$ the following equality holds

$$
2 \mathcal{T}_{\ell}+(\omega-2 \ell)\left\{(\omega-2 \ell-1) \mathcal{N}_{\ell}+4 \mathcal{M}_{\ell}\right\}=0 .
$$

Proof. By Theorem 3, we have $\underset{p_{1} \ldots p_{\omega+2}}{\operatorname{Sym}} \nabla_{p_{3} \ldots p_{\omega+2}} \operatorname{Ric}_{p_{1} p_{2}}=0$. Since the Ricci tensor is symmetric and its covariant derivatives commute, one can also rewrite this identity as follows:

$$
\sum_{1 \leq i<j \leq \omega+2} \nabla_{p_{1} \ldots \hat{p}_{i} \ldots \hat{p}_{j} \ldots p_{\omega+2}} \operatorname{Ric}_{p_{i} p_{j}}=0 .
$$

After contracting by $g^{p_{k+1} p_{k+2}} \ldots g^{p_{\omega+1} p_{\omega+2}}$, with $k=\omega+2-2 \ell \geq 2$ and $1 \leq \ell \leq \frac{\omega}{2}$, we obtain

$$
\sum_{1 \leq i<j \leq k} \nabla_{p_{1} \ldots \hat{p}_{i} \ldots \hat{p}_{j} \ldots p_{k}} \Delta^{\ell} \operatorname{Ric}_{p_{i} p_{j}}=0
$$

since we assumed $\mid \nabla^{\omega}$ scal $\mid=0$. Equality (5) is obtained by multiplying (6) with $\nabla_{p_{1} \ldots p_{k-2}} \Delta^{\ell} \operatorname{Ric}_{p_{k-1} p_{k}}$.

Lemma 5. For any $\ell \leq \frac{\omega}{2}$, the following inequality holds

$$
2 \mathcal{T}_{\ell}+(\omega-2 \ell) \mathcal{N}_{\ell} \geq 0 \text {. }
$$

Moreover, it follows that $\mathcal{T}_{\ell}+\mathcal{N}_{\ell}-2 \mathcal{M}_{\ell} \geq 0$.

Proof. For $\omega$ even and $\ell=\frac{\omega}{2}$, by definition $\mathcal{M}_{\ell}=\mathcal{N}_{\ell}=0$. Thus, the inequalities holds. For $\omega$ odd and $\ell=\frac{\omega-1}{2}$, by definition $\mathcal{N}_{\ell}=0$ and by (5), we have $\mathcal{T}_{\ell}+2 \mathcal{M}_{\ell}=$ 0 . Hence $\mathcal{M}_{\ell}$ is nonpositive, which proves the inequalities.

From now on, we assume that $\ell \leq \frac{\omega-2}{2}$ and set $k:=\omega-2 \ell+2 \geq 4$. We use the convention $\left(\begin{array}{l}p \\ q\end{array}\right)=0$ if $q>p$. Let $\Gamma(t)$ be the $k$-covariant tensor, depending on the real parameter $t$, whose components are given by

$$
\Gamma(t)_{p_{1} \ldots p_{k}}=\nabla_{p_{1} \ldots p_{k-2}} \Delta^{\ell} \operatorname{Ric}_{p_{k-1} p_{k}} t+\sum_{1 \leq i<j \leq k-2} \nabla_{p_{1} \ldots \hat{p}_{i} \ldots \hat{p}_{j} \ldots p_{k}} \Delta^{\ell} \operatorname{Ric}_{p_{i} p_{j}},
$$

The square norm of $\Gamma(t)$ is given by

$$
\begin{aligned}
\Gamma(t)_{p_{1} \ldots p_{k}} \Gamma(t)_{p_{1} \ldots p_{k}}=\mathcal{T}_{\ell} t^{2}+\left(\begin{array}{c}
k-2 \\
2
\end{array}\right) \mathcal{T}_{\ell}+2\left(\begin{array}{c}
k-2 \\
2
\end{array}\right) \mathcal{N}_{\ell} t+ \\
\quad\left(\begin{array}{c}
k-2 \\
2
\end{array}\right)\left(\begin{array}{c}
k-4 \\
2
\end{array}\right) \mathcal{N}_{\ell}+2\left(\begin{array}{c}
k-2 \\
2
\end{array}\right)(k-4) \mathcal{M}_{\ell}
\end{aligned}
$$


which is, with respect to $t$, a second degree nonnegative polynomial. Thus its discriminant is nonpositive, namely

$$
(k-2)(k-3)\left(\mathcal{N}_{\ell}\right)^{2}-\mathcal{T}_{\ell}\left\{2 \mathcal{T}_{\ell}+(k-4)(k-5) \mathcal{N}_{\ell}+4(k-4) \mathcal{M}_{\ell}\right\} \leq 0 .
$$

We substitute $\mathcal{M}_{\ell}$, using (5) and obtain

$$
(k-2)(k-3)\left(\frac{\mathcal{N}_{\ell}}{\mathcal{T}_{\ell}}\right)^{2}+2(k-4)\left(\frac{\mathcal{N}_{\ell}}{\mathcal{T}_{\ell}}\right)-\frac{4}{k-2} \leq 0,
$$

where we assumed that $\mathcal{T}_{\ell} \neq 0$ (otherwise the proof of the lemma is trivial, since all terms involved vanish). We conclude that $\frac{-2}{k-2} \leq\left(\frac{\mathcal{N}_{\ell}}{\mathcal{T}_{\ell}}\right) \leq \frac{2}{(k-2)(k-3)}$. This proves the first inequality, since $\omega \geq 2 \ell+2$. Substituting the value of $\mathcal{M}_{\ell}$, given by (5), in $\mathcal{T}_{\ell}+\mathcal{N}_{\ell}-2 \mathcal{M}_{\ell}$ and using the first inequality of the lemma yields the second one.

\section{Proof of Aubin's theorem}

We recall that $(M, g)$ denotes a compact Riemannian manifold of dimension $n$ and $x \in M$ is a fixed point. We assumed that $\operatorname{det}(g)=1+O\left(r^{N}\right)$, with $N$ sufficiently large, in a geodesic normal coordinate chart around $x$ and that $\left|\nabla^{\omega} \operatorname{scal}(x)\right|=0$. We use the following notation for the symmetrizations that occur in our computation:

$$
\begin{aligned}
\mathcal{S} & =\operatorname{Tr} \operatorname{Sym}_{K} \operatorname{Ric}_{a b}, & \mathcal{S}_{1} & =\operatorname{Tr} \operatorname{Sym}_{K} \nabla_{\text {Scal }}, \\
\mathcal{S}_{2} & =\operatorname{Tr} \operatorname{Sym}_{I} R_{i a b j} \cdot \nabla_{J} \operatorname{Ric}_{i j}, & \mathcal{S}_{3} & =\operatorname{Tr} \operatorname{Sym}_{I} \operatorname{Ric}_{a j} \cdot \nabla_{J} \operatorname{Ric}_{b j}, \\
\mathcal{S}_{4} & =\operatorname{Tr} \operatorname{Sym} \nabla_{I} R_{i a b j} \cdot \nabla_{J^{\prime}} \nabla_{i} \operatorname{Ric}_{c j}, & \mathcal{Q}(R)_{I J a b c d} & =\operatorname{Sym} \nabla_{I} R_{i a b j} \cdot \nabla_{J} R_{i c d j},
\end{aligned}
$$

where $I, J, J^{\prime}$ and $K$ denote multi-indices of length $\# K=2 \omega+2, \# I=\# J=\omega$, $\# J^{\prime}=\omega-1$ and the symmetrization is taken over all the indices which are not yet contracted. Note that $\mathcal{Q}(R)$ is a symmetric $(2 \omega+4)$-covariant tensor and $\mathcal{S}$, $\mathcal{S}_{1}, \ldots, \mathcal{S}_{4}$ are real numbers. Using this notation, the two-by-two contraction of the Hebey-Vaugon formula $(1)$ is $\mathcal{S}+C(\omega) \operatorname{Tr} \mathcal{Q}(R)=0$.

Before we start the computation, let us here illustrate the idea of our proof of Theorem 1. By (4), it is sufficient to show that $\Delta^{\omega+1}$ scal $<0$. The first step in the proof is to compute $\mathcal{S}$, which is the two-by-two contraction of the covariant derivatives of the Ricci tensor. When the order of the covariant derivatives is less or equal to $2 \omega+1$, we know that they commute (cf. Theorem 3). Therefore, the two-by-two contraction of the covariant derivatives of the Ricci tensor is, up to a positive integer, equal to either $\Delta^{\ell}$ scal or $\nabla \Delta^{\ell}$ scal for some nonnegative integer $\ell$ (cf. (2) and (3)). However in $\mathcal{S}$ and $\mathcal{S}_{1}$, there are $2 \omega+2$ covariant derivatives and in general they do not commute. Each time we commute two of them in $\mathcal{S}$ or $\mathcal{S}_{1}$, the Riemann curvature tensor occurs. By using the fact that for any nonnegative integer $k \leq \omega-1, \mid \nabla^{k}$ Riem $\mid=0$ and $\mid \nabla^{\omega}$ scal $\mid=0$, we show that the $2 \omega+2$ covariant derivatives of the scalar curvature in $\mathcal{S}_{1}$ commute and thus $\mathcal{S}_{1}=(2 \omega+2) ! \Delta^{\omega+1}$ scal. Using this fact, we prove that $\mathcal{S}$ is equal to the sum of a positive integer times $\Delta^{\omega+1}$ scal and a positive combination, denoted $\mathcal{J}$, of the terms $\mathcal{S}_{2}, \mathcal{S}_{3}$ and $\mathcal{S}_{4}$, which corresponds to the commutativity obstruction of the covariant derivatives in $\mathcal{S}$. By taking into account the formula $\mathcal{S}+C(\omega) \operatorname{Tr} \mathcal{Q}(R)=0$, it is sufficient to show that $\mathcal{J}+C(\omega) \operatorname{Tr} \mathcal{Q}(R)>0$. The second step is to compute the two-by-two contraction of $\mathcal{Q}(R)$. We determine all possible terms that occur when contracting all the Riemann tensor entries of $\mathcal{Q}(R)$ (i.e. in the definition of $\mathcal{Q}(R)$, they correspond to $a, b, c$ and $d)$. It turns out that $\operatorname{Tr} \mathcal{Q}(R)$ is a positive integer combination of 27 terms (without using Bianchi identities), denoted $\mathcal{A}_{k}$, where each $\mathcal{A}_{k}$ is a two-by-two contraction of a symmetric tensor of the same form as $\mathcal{Q}(R)$, but with contracted Riemann tensor entries. Further, we prove that all 
$\mathcal{A}_{k}$ 's are nonnegative and some of them are positive (they are summarized in Table 1). These 27 terms $\mathcal{A}_{k}$ are themselves a positive combination of the following 4 nonnegative terms $\left\{\mathcal{R}_{\ell}, \mathcal{T}_{\ell}, \mathcal{T}_{\ell}-2 \mathcal{M}_{\ell}+\mathcal{N}_{\ell}, \mathcal{T}_{\ell}-\mathcal{M}_{\ell}\right\}$, which are defined in Section 4. The third step is to do the same thing as in the second step for $\mathcal{J}$. Namely, we compute all possible terms occurring in the two-by-two contraction. We establish that $\mathcal{J}$ is also an integer (with positive and negative coefficients) combination of the following 3 terms $\left\{\mathcal{T}_{\ell}, \mathcal{M}_{\ell}, \mathcal{N}_{\ell}\right\}$. In the last step, one has to add the two combinations of $\operatorname{Tr} \mathcal{Q}(R)$ and $\mathcal{J}$ and check, using Lemma 5 and (5), that each kind of term, occurring in $\mathcal{J}+C(\omega) \operatorname{Tr} \mathcal{Q}(R)$, is nonnegative. We conclude by using the fact that $\mathcal{R}_{0}$ is positive, which holds by the definition of $\omega$.

Proposition 6. The following equality involving the above defined symmetrizations holds:

$$
\begin{aligned}
& \mathcal{S}=2(\omega+2)^{2}(2 \omega+2) ! \Delta^{\omega+1} \text { scal }+C(\omega)\left\{2(\omega+3)^{2}\left(\mathcal{S}_{2}+\mathcal{S}_{3}\right)+2 \omega(\omega+3) \mathcal{S}_{4}\right\}, \\
& \text { where } C(\omega)=(\omega+1)^{2}(\omega+2)^{2}(2 \omega+2) ![(\omega+3) !]^{-2}
\end{aligned}
$$

By Theorem 3, we have $\mathcal{S}+C(\omega) \operatorname{Tr} \mathcal{Q}(R)=0$, If we substitute $\mathcal{S}$ by its value given in Proposition 6 , we obtain the following equality.

Corollary 7. The following equality holds

$$
-\Delta^{\omega+1} \text { scal }=\frac{(\omega+1)^{2}}{2[(\omega+3) !]^{2}}\left\{\operatorname{Tr} \mathcal{Q}(R)+2(\omega+3)\left[(\omega+3)\left(\mathcal{S}_{2}+\mathcal{S}_{3}\right)+\omega \mathcal{S}_{4}\right]\right\}
$$

Remark 8. Note that the equality above is claimed by Aubin 1, p. 279], which in his notation, is written in the form $2(\omega+2)^{2} \operatorname{Tr} \operatorname{Sym} \nabla_{\alpha \beta k l} R+C(\omega) I=0$.

Proof of Proposition 6. In order to prove (7), we start by decomposing the symmetrization of the $(2 \omega+2)$-covariant derivatives of the Ricci tensor and then contract two-by-two as follows:

$$
\begin{gathered}
\mathcal{S}=2(\omega+2)\left\{\mathcal{S}_{1}+\operatorname{Tr}_{\substack{\sigma \in \mathfrak{S}(2 \omega+2) \\
1 \leq k \leq 2 \omega+2}} \nabla_{p_{\sigma(1)} \ldots p_{\sigma(k-1)} q p_{\sigma(k)} \ldots p_{\sigma(2 \omega+1)}} \operatorname{Ric}_{p_{\sigma(2 \omega+2)} q}\right\} \\
=2(\omega+2)\left\{\mathcal{S}_{1}+\operatorname{Tr}_{\substack{\sigma \in \mathfrak{S}(2 \omega+2) \\
\omega+1 \leq k \leq 2 \omega+2}}\left(1+\omega \delta_{k(\omega+1)}\right) \nabla_{p_{\sigma(1)} \ldots p_{\sigma(k-1)} q p_{\sigma(k)} \ldots p_{\sigma(2 \omega+1)}} \operatorname{Ric}_{p_{\sigma(2 \omega+2)} q}\right\},
\end{gathered}
$$

where $\delta_{i j}$ denotes the Kronecker delta. The last equality follows by using the fact that for any $k \leq \omega-1, \mid \nabla^{k}$ Riem $\mid=0$ (cf. Theorem 3 ), which implies that any two successive covariant derivatives of order at least $\omega+2$ and $\omega+3$ respectively, in the $(2 \omega+2)$-covariant derivatives of the Ricci tensor, commute. Namely, for each $1 \leq k \leq \omega+1$ the following equality holds

$$
\nabla_{p_{\sigma(1)} \ldots p_{\sigma(k-1)} q p_{\sigma(k)} \ldots p_{\sigma(2 \omega+1)}} \operatorname{Ric}_{p_{\sigma(2 \omega+2)} q}=\nabla_{p_{\sigma(1)} \ldots p_{\sigma(\omega)} q p_{\sigma(\omega+1)} \ldots p_{\sigma(2 \omega+1)}} \operatorname{Ric}_{p_{\sigma(2 \omega+2)} q} .
$$

In the last sum over $k$ in (9), we obtain for $k=2 \omega+2$ a multiple of $\mathcal{S}_{1}$, by applying the contracted second Bianchi identity (A-1):

$$
\operatorname{Tr} \sum_{\sigma \in \mathfrak{S}(2 \omega+2)} \nabla_{p_{\sigma(1)} \ldots p_{\sigma(2 \omega+1)} q} \operatorname{Ric}_{p_{\sigma(2 \omega+2)} q}=\frac{1}{2} \mathcal{S}_{1} .
$$

For all the other terms in the last sum over $k$ in (9) we move the index $q$ to the right in order to get it as last index of the derivatives and then apply again (10). Thus, for each $\omega+1 \leq k \leq 2 \omega+2$, we obtain a term equal to $\frac{1}{2} \mathcal{S}_{1}$. Summing up, we 
obtain that $\mathcal{S}_{1}$ occurs in $\mathcal{S}$ with coefficient $2(\omega+2)\left[1+\frac{1}{2} \sum_{k=\omega+1}^{2 \omega+2}\left(1+\omega \delta_{k(\omega+1)}\right)\right]=$ $2(\omega+2)^{2}$.

In the terms of the last sum in (9), each permutation of $q$ to the right gives rise to new terms involving the Riemannian curvature tensor. More precisely, for each $\omega+1 \leq k \leq 2 \omega+1$, the following formula holds:

$$
\begin{aligned}
& \nabla_{p_{\sigma(1)} \ldots p_{\sigma(k-1)} q p_{\sigma(k)} \ldots p_{\sigma(2 \omega+1)}} \operatorname{Ric}_{p_{\sigma(2 \omega+2)} q}= \\
& \nabla_{p_{\sigma(1)} \ldots p_{\sigma(k-1)}} p_{\sigma(k)} q p_{\sigma(k+1)} \ldots p_{\sigma(2 \omega+1)} \operatorname{Ric}_{p_{\sigma(2 \omega+2)} q}+ \\
& \nabla_{p_{\sigma(1)} \ldots p_{\sigma(k-1)}}\left\{\sum_{j=k+1}^{2 \omega+1} R_{p p_{\sigma(j)} p_{\sigma(k)} q} \nabla_{p_{\sigma(k+1)} \ldots \hat{p}_{\sigma(j)} \ldots p_{\sigma(2 \omega+1)}} \nabla_{p} \operatorname{Ric}_{p_{\sigma(2 \omega+2)} q}+\right. \\
&\left.R_{p p_{\sigma(2 \omega+2)} p_{\sigma(k)} q} \nabla_{p_{\sigma(k+1)} \ldots p_{\sigma(2 \omega+1)}} \operatorname{Ric}_{p q}+\operatorname{Ric}_{p_{\sigma(k)} p} \nabla_{p_{\sigma(k+1)} \ldots p_{\sigma(2 \omega+1)}} \operatorname{Ric}_{p_{\sigma(2 \omega+2)} p}\right\}
\end{aligned}
$$

where we used the fact that the covariant derivatives commute up to order $2 \omega+1$ (cf. Theorem 3). Note that for $k=2 \omega+1$, the sum over $j$ in (11) is empty, meaning that the corresponding terms do not occur in this case.

In the first term of (11), we continue to move the index $q$ to the right, using repeatedly the same formula until $q$ attains the last position in the indices of the covariant derivatives. For the remaining terms in (11), we first compute, using the Leibniz rule, their covariant derivatives. For example, for the second type of term, we have for each $\omega+1 \leq k \leq 2 \omega+1$ :

$$
\begin{aligned}
& \nabla_{p_{\sigma(1)} \ldots p_{\sigma(k-1)}}\left(R_{p p_{\sigma(2 \omega+2)} p_{\sigma(k)} q} \cdot \nabla_{p_{\sigma(k+1)} \ldots p_{\sigma(2 \omega+1)}} \operatorname{Ric}_{p q}\right)= \\
& \sum_{S \subseteq\left\{p_{\sigma(1)}, \ldots, p_{\sigma(k-1)}\right\}, \# S=\omega}\left(\nabla_{S} R_{p p_{\sigma(2 \omega+2)} p_{\sigma(k)} q}\right)\left(\nabla_{\left\{p_{\sigma(1)} \ldots p_{\sigma(k-1)}\right\} \backslash S} \nabla_{p_{\sigma(k+1)} \ldots p_{\sigma(2 \omega+1)}} \operatorname{Ric}_{p q}\right) .
\end{aligned}
$$

Taking now in the last equality the sum over all permutations $\sigma \in \mathfrak{S}(2 \omega+2)$ and contracting two-by-two, we obtain for each $\omega+1 \leq k \leq 2 \omega+1$ :

$$
\operatorname{Tr} \sum_{\sigma \in \mathfrak{S}(2 \omega+2)} \nabla_{p_{\sigma(1)} \ldots, p_{\sigma(k-1)}}\left(R_{p p_{\sigma(2 \omega+2)} p_{\sigma(k)} q} \nabla_{p_{\sigma(k+1)} \ldots p_{\sigma(2 \omega+1)}} \operatorname{Ric}_{p q}\right)=\left(\begin{array}{c}
k-1 \\
\omega
\end{array}\right) \mathcal{S}_{2} .
$$

After iterating the formula (11) sufficiently many times in order to transform all terms occurring in the last sum in (9) into terms having the index $q$ as the last index of the covariant derivates, we can compute as follows the coefficient of $\mathcal{S}_{2}$ in $\mathcal{S}$ :

$2(\omega+2) \sum_{k=\omega+1}^{2 \omega+1}\left\{\left(1+\omega \delta_{(\omega+1) k}\right) \sum_{j=k-1}^{2 \omega}\left(\begin{array}{l}j \\ \omega\end{array}\right)\right\}=2(\omega+2) \sum_{k=\omega}^{2 \omega}(k+1)\left(\begin{array}{l}k \\ \omega\end{array}\right)=2 C(\omega)(\omega+3)^{2}$.

The proof of the last equality is given in Appendix A.2. Applying the same argument as above to the last type of term in (11), yields that $\mathcal{S}_{3}$ occurs with the same multiplicity in $\mathcal{S}$. Now, we consider the remaining term in (11). For any $\omega+1 \leq k \leq 2 \omega$, we have 


$$
\begin{gathered}
\nabla_{p_{\sigma(1)} \ldots p_{\sigma(k-1)}}\left(\sum_{j=k+1}^{2 \omega+1} R_{p p_{\sigma(j)} p_{\sigma(k)} q} \nabla_{p_{\sigma(k+1)} \ldots \hat{p}_{\sigma(j)} \ldots p_{\sigma(2 \omega+1)}} \nabla_{p} \operatorname{Ric}_{p_{\sigma(2 \omega+2)} q}\right)= \\
\sum_{j=k+1}^{2 \omega+1} \sum_{\substack{S \subseteq\left\{p_{\sigma(1)} \ldots p_{\sigma(k-1)}\right\} \\
\# S=\omega}} \nabla_{S} R_{p p_{\sigma(j)} p_{\sigma(k)} q} \nabla_{\left\{p_{\sigma(1)} \ldots p_{\sigma(2 \omega+1)}\right\} \backslash\left(S \cup\left\{p_{\sigma(k)}, p_{\sigma(j)}\right\}\right)} \nabla_{p} \operatorname{Ric}_{p_{\sigma(2 \omega+2)} q} .
\end{gathered}
$$

Taking in this last equality the sum over all permutations $\sigma \in \mathfrak{S}(2 \omega+2)$ and contracting two-by-two, we obtain for each $\omega+1 \leq k \leq 2 \omega$ :

$$
\begin{array}{r}
\operatorname{Tr} \sum_{\sigma \in \mathfrak{S}(2 \omega+2)} \nabla_{p_{\sigma(1)} \ldots p_{\sigma(k-1)}} \sum_{j=k+1}^{2 \omega+1} R_{p p_{\sigma(j)} p_{\sigma(k)} q} \nabla_{p_{\sigma(k+1)} \ldots \hat{p}_{\sigma(j)} \ldots p_{\sigma(2 \omega+1)}} \nabla_{p} \operatorname{Ric}_{p_{\sigma(2 \omega+2)} q}= \\
\sum_{j=k+1}^{2 \omega+1}\left(\begin{array}{c}
k-1 \\
\omega
\end{array}\right) \mathcal{S}_{4}=(2 \omega+1-k)\left(\begin{array}{c}
k-1 \\
\omega
\end{array}\right) \mathcal{S}_{4}
\end{array}
$$

As above, in order to find the coefficient of $\mathcal{S}_{4}$ in $\mathcal{S}$, we combine (9), (12) and we obtain

$$
2(\omega+2) \sum_{k=\omega+1}^{2 \omega}\left\{\left(1+\omega \delta_{(\omega+1) k}\right) \sum_{j=k-1}^{2 \omega}(2 \omega-j)\left(\begin{array}{c}
j \\
\omega
\end{array}\right)\right\}=2 \omega(\omega+3) C(\omega),
$$

The proof of the last equality is given in Appendix A.2. To finish the proof of the proposition, it remains to show that $\mathcal{S}_{1}=(2 \omega+2) ! \Delta^{\omega+1}$ scal . In fact, when commuting any two successive covariant derivatives of the $(2 \omega+2)$-covariant derivatives of the scalar curvature, there are curvature terms occurring. These are all of the form $\nabla^{\alpha}$ Riem $\nabla^{\beta}$ scal, with $\alpha+\beta=2 \omega$. By Theorem 3, we know that for each $k \leq \omega-1$, we have $\mid \nabla^{k}$ Riem $|=| \nabla^{k}$ scal $\mid=0$. Since we assumed that $\mid \nabla^{\omega}$ scal $\mid=0$, it follows that the $(2 \omega+2)$-covariant derivatives of the scalar curvature commute.

We recall the definition of the symmetric $(2 \omega+4)$-covariant tensor $\mathcal{Q}(R)$, whose components $\mathcal{Q}(R)_{p_{1} \ldots p_{2 \omega+4}}$ are given by:

$$
\sum_{\sigma \in \mathfrak{S}(2 \omega+4)} \nabla_{p_{\sigma(1)} \ldots p_{\sigma(\omega)}} R_{i p_{\sigma(\omega+1)} p_{\sigma(\omega+2)} j} \cdot \nabla_{p_{\sigma(\omega+3)} \cdots p_{\sigma(2 \omega+2)}} R_{i p_{\sigma(2 \omega+3)} p_{\sigma(2 \omega+4)} j}
$$

Our purpose is to show that $\operatorname{Tr} \mathcal{Q}(R)$ is a positive combination of nonnegative terms. In order to define these terms, we need first to introduce some new notation. For all nonnegative integers $\ell$ and $\beta$, satisfying $0 \leq \beta \leq \omega$ and $0 \leq \ell \leq \frac{\omega-\beta}{2}$,

$$
\begin{aligned}
& \mathcal{T}_{\ell}^{\omega-\beta-2 \ell}:=\operatorname{Tr} \operatorname{Sym}_{I \cup J} \nabla_{I} \nabla_{K} \Delta^{\ell} \operatorname{Ric}_{i j} \nabla_{J} \nabla_{K} \operatorname{Ric}_{i j}, \\
& \mathcal{T}_{1,1}^{\omega-2}:=\operatorname{Tr} \underset{I^{\prime \prime} \cup J^{\prime \prime}}{\operatorname{Sym}_{I^{\prime \prime}}} \nabla_{\operatorname{Ric}_{i j}} \nabla_{J^{\prime \prime}} \Delta \operatorname{Ric}_{i j},
\end{aligned}
$$

where here $I, J, I^{\prime \prime}, J^{\prime \prime}$ and $K$ are multi-indices of cardinality $\omega-\beta-2 \ell, \omega-\beta$, $\omega-2, \omega-2$ and $\beta$ respectively. In other words, $\mathcal{T}_{\ell}^{\omega-\beta-2 \ell}$ is obtained by taking the product of $(\omega-2 \ell)$-covariant derivatives of $\Delta^{\ell}$ Ric with $\omega$-covariant derivatives of Ric, where $\beta$ covariant derivatives of both factors are already contracted to each other and by contracting two-by-two the symmetrization over the remaining covariant derivatives.

Set $\mathcal{T}^{\omega-\beta}=\mathcal{T}_{0}^{\omega-\beta}$. Note that $\mathcal{T}_{\ell}^{0}=(2 \ell) ! \mathcal{T}_{\ell}$ (cf. Section 4 for the definition of $\left.\mathcal{T}_{\ell}\right)$. The terms $\mathcal{R}_{\ell}^{\omega-\beta-2 \ell}, \mathcal{M}_{\ell}^{\omega-\beta-2 \ell}, \mathcal{N}_{\ell}^{\omega-\beta-2 \ell}, \mathcal{R}^{\omega-\beta}, \mathcal{M}^{\omega-\beta}$ and $\mathcal{N}^{\omega-\beta}$ are defined 
in the same way as $\mathcal{T}_{\ell}^{\omega-\beta-2 \ell}$ and $\mathcal{T}^{\omega-\beta}$. Now we show that these terms are integer combinations of $\mathcal{R}_{\ell}, \mathcal{T}_{\ell}, \mathcal{M}_{\ell}$ and $\mathcal{N}_{\ell}$. First, we note that

$$
\begin{aligned}
& \mathcal{T}^{\omega-\beta}=\operatorname{Tr} \operatorname{Sym}_{I} \nabla_{K} \operatorname{Ric}_{i j} \nabla_{J} \nabla_{K} \operatorname{Ric}_{i j} \\
&=2(\omega-\beta) \operatorname{Tr} \operatorname{Sym}\left\{(\omega-\beta) \nabla_{I^{\prime} K a} \operatorname{Ric}_{i j} \nabla_{J K a} \operatorname{Ric}_{i j}+(\omega-\beta-1) \nabla_{I^{\prime \prime} K a a} \operatorname{Ric}_{i j} \nabla_{J K} \operatorname{Ric}_{i j}\right\} \\
&=2(\omega-\beta)\left\{(\omega-\beta) \mathcal{T}^{\omega-\beta-1}+(\omega-\beta-1) \mathcal{T}_{1}^{\omega-\beta-2}\right\} .
\end{aligned}
$$

It follows that for any $2 \leq \gamma \leq \omega$, we have

$$
\mathcal{T}^{\gamma}=2 \gamma(\gamma-1) \mathcal{T}_{1}^{\gamma-2}+2 \gamma^{2} \mathcal{T}^{\gamma-1}
$$

Similarly, we obtain the following formulas:

$$
\begin{aligned}
& \mathcal{T}_{1}^{\gamma-2}=2 \gamma(\gamma-1) \mathcal{T}_{1}^{\gamma-3}+2(\gamma-3)(\gamma-1) \mathcal{T}_{2}^{\gamma-4}, \\
& \mathcal{T}_{1}^{\gamma-2}=2(\gamma-1)(\gamma-2) \mathcal{T}_{1}^{\gamma-3}+2(\gamma-1)^{2} \mathcal{T}_{1,1}^{\gamma-2} .
\end{aligned}
$$

Using (13), (14) and (15), we prove, by induction on $\gamma$, the following formulas

$$
\begin{aligned}
& \mathcal{T}^{\gamma}=\sum_{\ell=0}^{\left[\frac{\gamma}{2}\right]} d_{\ell}^{\gamma} \mathcal{T}_{\ell}, \text { for all } 0 \leq \gamma \leq \omega ; \quad \mathcal{T}_{1}^{\gamma-2}=\sum_{\ell=1}^{\left[\frac{\gamma}{2}\right]} e_{\ell-1}^{\gamma} \mathcal{T}_{\ell}, \text { for all } 2 \leq \gamma \leq \omega, \\
& \mathcal{T}_{1,1}^{\gamma-2}=\sum_{\ell=1}^{\left[\frac{\gamma}{2}\right]} d_{\ell-1}^{\gamma-2} \mathcal{T}_{\ell}, \text { for all } 2 \leq \gamma \leq \omega,
\end{aligned}
$$

where $d_{\ell}^{\gamma}:=\frac{2^{\gamma-2 \ell}(\gamma !)^{3}}{(\gamma-2 \ell) !(\ell !)^{2}}$ and $e_{\ell}^{\gamma}:=\frac{2^{\gamma-2 \ell-2} \gamma !(\gamma-1) !(\gamma-2) !}{(\gamma-2 \ell-2) !(\ell+1) ! \ell !}$. Note that the equalities (13) and (16) hold also for $\mathcal{R}^{\gamma}, \mathcal{M}^{\gamma-1}$ and $\mathcal{N}^{\gamma-2}$.

Claim 9. $\operatorname{Tr} \mathcal{Q}(R)=\sum_{k=1}^{27} u_{k} \mathcal{A}_{k}$ and $\mathcal{A}_{k}=\mathcal{B}_{k}$ for any $1 \leq k \leq 27$, where the terms $\mathcal{A}_{k}, \mathcal{B}_{k}$ and the coefficients $u_{k}$ are defined in Table 1.

Each term $\mathcal{A}_{k}$ of the second column denotes a two-by-two contraction of a symmetric tensor, where the symmetrization is taken over all the indices which are not already contracted. In the fourth column, we define the terms $\mathcal{B}_{k}$. We prove using the two Bianchi identities that $\mathcal{A}_{k}=\mathcal{B}_{k}$ (cf. Appendix A.1). The coefficients $u_{k}$ are given in terms of the following positive integers:

$$
\begin{array}{ll}
c_{1}:=(2 \omega+4)(2 \omega+2), & c_{3}:=2 \omega^{2}(\omega-1) c_{1}, \\
c_{2}:=2 \omega^{3} c_{1}, & c_{5}:=4 \omega^{2}(\omega-1)^{2}(\omega-2)(\omega-3) c_{1},
\end{array}
$$

Let us recall that the covariant derivates of the Riemann curvature tensor already commute ( $c f$. Theorem 3). This allows us to determine all types of terms that are obtained after taking the two-by-two contractions of $\mathcal{Q}(R)$ involving the indices that occur directly in the Riemann curvature tensor, namely of the following kind:

$$
g^{a \alpha} g^{b \beta} g^{c \gamma} g^{d \delta} \nabla_{I} R_{i a b j} \cdot \nabla_{J} R_{i c d j}
$$

where $\{a, b, c, d\} \cup I \cup J=\left\{p_{1}, \ldots, p_{2 \omega+4}\right\}$ is an arbitrary partition of $\left\{p_{1}, \ldots, p_{2 \omega+4}\right\}$ into subsets of cardinality 4 , respectively $\# I=\# J=\omega$ and $\{\alpha, \beta, \gamma, \delta\}$ is any subset of 4 indices of $\left\{p_{1}, \ldots, p_{2 \omega+4}\right\}$, such that $\alpha \neq a, \beta \neq b, \gamma \neq c$ and $\delta \neq d$.

After taking into account the commutativity of the product, we identify 27 types of terms like (18), which are denoted by $\mathcal{A}_{k}$ and listed in the second column of Table 1. Moreover, if $I$ is a multi-index of length $\omega$, then $I^{\prime}$ denotes a multi-index of length $\omega-1, I^{\prime \prime}$ a multi-index of length $\omega-2$ and so on. We also note that each of the sums over the contraction indices $a, b, c, d$ runs from 1 to $n$ and that not all 27 types occur for $\omega \leq 3$, for instance for $\omega=2$ the terms $\mathcal{A}_{19}, \ldots, \mathcal{A}_{23}$ in the table do not occur.

We further compute the multiplicity of each such term of type (18) in the twoby-two contraction of $\mathcal{Q}(R)$. We write it down in the third column of Table 1 . 


\begin{tabular}{|c|c|c|c|}
\hline$k$ & $\mathcal{A}_{k}$ & $u_{k}$ & $\mathcal{B}_{k}$ \\
\hline 1 & $\overline{\operatorname{Tr} \operatorname{Sym} \nabla_{I} \mathrm{Ric}_{i j} \nabla_{J} \mathrm{Ric}_{i j}}$ & $c_{1}$ & $\mathcal{T}^{\omega}$ \\
\hline 2 & $-\operatorname{Tr} \operatorname{Sym}_{I^{\prime} c} \operatorname{Ric}_{i j} \nabla_{J^{\prime} b} R_{i b c j}$ & $2 c_{2}$ & $\mathcal{T}^{\omega-1}-\mathcal{M}^{\omega-1}$ \\
\hline 3 & $-\operatorname{Tr} \operatorname{Sym}_{I} \operatorname{Ric}_{i j} \nabla_{J^{\prime \prime} b c} R_{i b c j}$ & $2 c_{3}$ & $\mathcal{T}_{1}^{\omega-2}$ \\
\hline 4 & $-\operatorname{Tr} \operatorname{Sym}_{I^{\prime} b} \operatorname{Ric}_{i j} \nabla_{J^{\prime} c} R_{i b c j}$ & $2 c_{2}$ & $\mathcal{B}_{2}$ \\
\hline 5 & $-\operatorname{Tr} \operatorname{Sym}_{I^{\prime \prime} b c} \operatorname{Ric}_{i j} \nabla_{J} R_{i b c j}$ & $2 c_{3}$ & $\sum_{\ell=0}^{\left[\frac{\omega-2}{2}\right]} e_{\ell}^{\omega}\left(\mathcal{T}_{\ell}-2 \mathcal{M}_{\ell}+\mathcal{N}_{\ell}\right)$ \\
\hline 6 & $\operatorname{Tr} \operatorname{Sym} \nabla_{I} R_{i a b j} \nabla_{J} R_{i a b j}$ & $c_{1}$ & $\mathcal{R}^{\omega}$ \\
\hline 7 & $\operatorname{Tr} \operatorname{Sym} \nabla_{I^{\prime} b} R_{i a b j} \nabla_{J^{\prime} c} R_{i a c j}$ & $c_{2}$ & $2 \mathcal{B}_{2}$ \\
\hline 8 & $\operatorname{Tr} \operatorname{Sym} \nabla_{I^{\prime \prime} c b} R_{i a b j} \nabla_{J} R_{i a c j}$ & $2 c_{3}$ & $2 \mathcal{B}_{5}$ \\
\hline 9 & $\operatorname{Tr} \operatorname{Sym} \nabla_{I^{\prime} c} R_{i a b j} \nabla_{J^{\prime} b} R_{i a c j}$ & $c_{2}$ & $\frac{1}{2} \mathcal{R}^{\omega-1}$ \\
\hline 10 & $\operatorname{Tr} \operatorname{Sym} \nabla_{I} R_{i a b j} \nabla_{J} R_{i b a j}$ & $c_{1}$ & $\frac{1}{2} \mathcal{B}_{6}$ \\
\hline 11 & $\operatorname{Tr} \operatorname{Sym} \nabla_{I^{\prime} b} R_{i a b j} \nabla_{J^{\prime} c} R_{i c a j}$ & $2 c_{2}$ & $\mathcal{B}_{2}$ \\
\hline 12 & $\operatorname{Tr} \operatorname{Sym} \nabla_{I^{\prime \prime} b c} R_{i a b j} \nabla_{J} R_{i c a j}$ & $2 c_{3}$ & $\mathcal{B}_{5}$ \\
\hline 13 & $\operatorname{Tr} \operatorname{Sym} \nabla_{I^{\prime} c} R_{i a b j} \nabla_{J^{\prime} b} R_{i c a j}$ & $2 c_{2}$ & $\frac{1}{2} \mathcal{B}_{9}$ \\
\hline 14 & $\operatorname{Tr} \operatorname{Sym} \nabla_{I} R_{i a b j} \nabla_{J^{\prime \prime} b c} R_{i c a j}$ & $2 c_{3}$ & $\mathcal{B}_{5}$ \\
\hline 15 & $\operatorname{Tr} \operatorname{Sym} \nabla_{I} R_{i a b j} \nabla_{J^{\prime \prime} a c} R_{i c b j}$ & $2 c_{3}$ & $2 \mathcal{B}_{5}$ \\
\hline 16 & $\operatorname{Tr} \operatorname{Sym} \nabla_{I^{\prime} c} R_{i a b j} \nabla_{J^{\prime} a} R_{i c b j}$ & $c_{2}$ & $\mathcal{B}_{9}$ \\
\hline 17 & $\operatorname{Tr} \operatorname{Sym} \nabla_{I^{\prime} a} R_{i a b j} \nabla_{J^{\prime} c} R_{i c b j}$ & $c_{2}$ & $2 \mathcal{B}_{2}$ \\
\hline 18 & $\operatorname{Tr} \operatorname{Sym} \nabla_{I^{\prime \prime} a b} R_{i a b j} \nabla_{J^{\prime \prime} c d} R_{i c d j}$ & $c_{4}$ & $\mathcal{T}_{1,1}^{\omega-2}$ \\
\hline 19 & $\operatorname{Tr} \operatorname{Sym} \nabla_{I^{\prime \prime \prime \prime} a b c d} R_{i a b j} \nabla_{J} R_{i c d j}$ & $2 c_{5}$ & $\sum_{\ell=0}^{\left[\frac{\omega-2}{2}\right]} \frac{\ell e_{\ell}^{\omega}\left(\mathcal{T}_{\ell}-2 \mathcal{M}_{\ell}+\mathcal{N}_{\ell}\right)}{(\omega-1)(\omega-2)(\omega-3)}$ \\
\hline 20 & $\operatorname{Tr} \operatorname{Sym} \nabla_{I^{\prime \prime \prime}{ }_{a b c} R_{i a b j}} \nabla_{J^{\prime} d} R_{i c d j}$ & $2 c_{6}$ & $\mathcal{T}_{1}^{\omega-3}-\mathcal{M}_{1}^{\omega-3}$ \\
\hline 21 & $\operatorname{Tr} \operatorname{Sym} \nabla_{I^{\prime \prime \prime} a b d} R_{i a b j} \nabla_{J^{\prime} c} R_{i c d j}$ & $2 c_{6}$ & $\mathcal{B}_{20}$ \\
\hline 22 & $\operatorname{Tr} \operatorname{Sym} \nabla_{I^{\prime} c} R_{i a b j} \nabla_{J^{\prime \prime \prime} d a b} R_{i c d j}$ & $2 c_{6}$ & $\sum_{\ell=0}^{\left[\frac{\omega-3}{2}\right]} e_{\ell}^{\omega-1}\left(\mathcal{T}_{\ell}-2 \mathcal{M}_{\ell}+\mathcal{N}_{\ell}\right)$ \\
\hline 23 & $\operatorname{Tr} \operatorname{Sym} \nabla_{I^{\prime} d} R_{i a b j} \nabla_{J^{\prime \prime \prime} c a b} R_{i c d j}$ & $2 c_{6}$ & $\mathcal{B}_{22}$ \\
\hline 24 & $\operatorname{Tr} \operatorname{Sym} \nabla_{I^{\prime \prime} c d} R_{i a b j} \nabla_{J^{\prime \prime} a b} R_{i c d j}$ & $c_{4}$ & $\frac{1}{2} \mathcal{R}^{\omega-2}$ \\
\hline 25 & $\left.\operatorname{Tr} \operatorname{Sym} \nabla_{I^{\prime \prime} d a} R_{i a b j} \nabla_{J^{\prime \prime} b c} R_{i c d j}\right)$ & $c_{4}$ & $\mathcal{T}^{\omega-2}-\mathcal{M}^{\omega-2}$ \\
\hline 26 & $\operatorname{Tr} \operatorname{Sym} \nabla_{I^{\prime \prime} c a} R_{i a b j} \nabla_{J^{\prime \prime} b d} R_{i c d j}$ & $2 c_{4}$ & $\mathcal{T}^{\omega-2}-2 \mathcal{M}^{\omega-2}+\mathcal{N}^{\omega-2}$ \\
\hline 27 & $\operatorname{Tr} \operatorname{Sym} \nabla_{I^{\prime \prime} c b} R_{i a b j} \nabla_{J^{\prime \prime} a d} R_{i c d j}$ & $c_{4}$ & $\mathcal{B}_{25}$ \\
\hline
\end{tabular}

TABLE 1.

Let us here only illustrate how we determined the multiplicity for one of the terms. For example, the third term $\mathcal{A}_{3}=\operatorname{Tr} \operatorname{Sym} \nabla_{I} R_{i a a j} \cdot \nabla_{J^{\prime \prime} b c} R_{i b c j}$ has multiplicity equal to $2 c_{3}$ as follows. For the choice of the three indices $a, b$ and $c$ among the $\omega+2$ pairs of distinct indices over which the contraction is done we have $\omega(\omega+1)(\omega+2)$ possibilities, and for each such fixed choice we may permute the two indices of each such pair, thus multiplying the coefficient by $2^{3}$. Further we have to multiply by $\omega(\omega-1)$, since we may choose for $b$ and $c$ any two indices (whose order also counts) of the multi-index of length $\omega$ corresponding to the covariant derivatives, because these commute, as remarked above. The remaining $2 \omega-2$ indices in $I$ and $J^{\prime \prime}$ may be permuted arbitrarily. One still has to take into account that the two factors of each term commute and since the factors of this term are not symmetric, its coefficient has to be doubled. Concluding, we obtain the claimed multiplicity. For all the other types of terms in the table the multiplicity is computed similarly. 
To verify that we obtained all the possible terms, we check, by summing up the multiplicities, that

$$
(2 \omega) !\left(u_{1}+u_{6}+u_{10}\right)+(2 \omega-2) ! \sum_{\substack{k=2 \\ k \notin\{6,10\}}}^{17} u_{k}+(2 \omega-4) ! \sum_{k=18}^{27} u_{k}=(2 \omega+4) ! .
$$

Using the two Bianchi identities, we show that for any $1 \leq k \leq 27, \mathcal{A}_{k}=\mathcal{B}_{k}$ (the details of the computation can be found in Appendix A.1). This finishes the proof of the claim. As a consequence, we obtain the first part of the following result.

Proposition 10. The following equality holds: $\operatorname{Tr} \mathcal{Q}(R)=\sum_{k=1}^{27} u_{k} \mathcal{B}_{k}$, where the $u_{k}$ 's are positive integer coefficients and $\mathcal{B}_{k}$ 's are nonnegative terms, defined in Table 1. Furthermore, it follows that

$$
\begin{gathered}
\operatorname{Tr} \mathcal{Q}(R)>(2 \omega+4)(2 \omega+2)\left\{\mathcal{T}^{\omega}+4 \omega^{2}(\omega-1) \mathcal{T}_{1}^{\omega-2}+4 \omega^{3}(\omega-1)^{3} \mathcal{T}_{1,1}^{\omega-2}+\right. \\
\left.4 \omega^{3}(\omega+4)\left(\mathcal{T}^{\omega-1}-\mathcal{M}^{\omega-1}\right)+8 \omega^{3}(\omega-1)^{2}(\omega-2)\left(\mathcal{T}_{1}^{\omega-3}-\mathcal{M}_{1}^{\omega-3}\right)+4 \omega^{2}(\omega-1)(\omega+7) \mathcal{B}_{5}\right\} .
\end{gathered}
$$

Proof. We still have to show that the $\mathcal{B}_{k}$ 's are nonnegative. For this, we further contract two-by-two the remaining indices occurring in the covariant derivatives in each of the $\mathcal{B}_{k}$ 's. Since the covariant derivatives of order less or equal to $\omega$ occurring in the terms $\mathcal{B}_{k}$ commute, one establishes that for any $1 \leq k \leq 27, \mathcal{B}_{k}=$ $\sum_{\ell=0}^{\left[\frac{\omega}{2}\right]} \alpha_{k \ell} \mathcal{B}_{k \ell}$, where $\alpha_{k \ell}$ are nonnegative integers representing the multiplicities and

$$
\mathcal{B}_{k \ell} \in\left\{\mathcal{R}_{\ell}, \mathcal{T}_{\ell}, \mathcal{T}_{\ell}-2 \mathcal{M}_{\ell}+\mathcal{N}_{\ell}, \mathcal{T}_{\ell}-\mathcal{M}_{\ell}\right\}
$$

due to equalities (16), (17), which hold for $\mathcal{R}^{\omega}, \mathcal{T}^{\omega}, \mathcal{M}^{\omega-1}$ and $\mathcal{N}^{\omega-2}$. Clearly, $\mathcal{R}_{\ell}$ and $\mathcal{T}_{\ell}$ are nonnegative. By Lemma $5, \mathcal{T}_{\ell}-2 \mathcal{M}_{\ell}+\mathcal{N}_{\ell}$ are nonnegative. The terms $\mathcal{T}_{\ell}-\mathcal{M}_{\ell}$ are also nonnegative, as it follows by writing down that the square norm of the $k$-covariant tensor $\nabla_{K c} \Delta^{\ell} \operatorname{Ric}_{a b}-\nabla_{K a} \Delta^{\ell} \operatorname{Ric}_{b c}$ is nonnegative, where $K$ is a multi-index $(\omega-2 \ell-1)$-tuple. Therefore the $\mathcal{B}_{k}$ 's are nonnegative.

Substituting in the equality $\operatorname{Tr} \mathcal{Q}(R)=\sum_{k=1}^{27} u_{k} \mathcal{B}_{k}$, proven in Claim 9 , the values of $\mathcal{B}_{k}$ and $u_{k}$ from Table 1 , we obtain

$$
\begin{gathered}
\operatorname{Tr} \mathcal{Q}(R)=(2 \omega+4)(2 \omega+2)\left\{\frac{3}{2} \mathcal{R}^{\omega}+3 \omega^{3} \mathcal{R}^{\omega-1}+2 \omega^{3}(\omega-1)^{3} \mathcal{R}^{\omega-2}+\right. \\
\mathcal{T}^{\omega}+4 \omega^{2}(\omega-1) \mathcal{T}_{1}^{\omega-2}+4 \omega^{3}(\omega-1)^{3} \mathcal{T}_{1,1}^{\omega-2}+20 \omega^{3}\left(\mathcal{T}^{\omega-1}-\mathcal{M}^{\omega-1}\right)+ \\
8 \omega^{3}(\omega-1)^{3}\left(\mathcal{T}^{\omega-2}-\mathcal{M}^{\omega-2}\right)+16 \omega^{3}(\omega-1)^{2}(\omega-2)\left(\mathcal{T}_{1}^{\omega-3}-\mathcal{M}_{1}^{\omega-3}\right)+ \\
\left.28 \omega^{2}(\omega-1) \mathcal{B}_{5}+8 \omega^{2}(\omega-1)^{2}\left[(\omega-2)(\omega-3) \mathcal{B}_{19}+2 \omega(\omega-2) \mathcal{B}_{22}+\omega(\omega-1) \mathcal{B}_{26}\right]\right\} .
\end{gathered}
$$

It order to prove the inequality stated in the proposition, we use the following equalities:

$$
\begin{gathered}
\mathcal{T}^{\omega-2}-\mathcal{M}^{\omega-2}=\frac{1}{2(\omega-1)^{2}}\left(\mathcal{T}^{\omega-1}-\mathcal{M}^{\omega-1}\right)-\frac{\omega-2}{\omega-1}\left(\mathcal{T}_{1}^{\omega-3}-\mathcal{M}_{1}^{\omega-3}\right) \\
\mathcal{B}_{26}=\frac{1}{2(\omega-1)^{2}} \mathcal{B}_{5}-\frac{\omega-2}{\omega-1} \mathcal{B}_{22}
\end{gathered}
$$

where the first one holds by (13) and the second one holds from the definition of $\mathcal{B}_{5}, \mathcal{B}_{22}$ and $\mathcal{B}_{26}$. Substituting (20) and (21) in (19) and using the fact that by the definition of $\omega, \mathcal{R}_{0}:=\mid \nabla^{\omega}$ Riem $\left.\right|^{2}$ is positive, we obtain the desired strict inequality. 
Remark 11. There is another method to prove the equality $\operatorname{Tr} \mathcal{Q}(R)=\sum_{k=1}^{27} u_{k} \mathcal{B}_{k}$ of Proposition 10. One may contract the 4 entries of the Riemann tensor in $\mathcal{Q}(R)$ one by one and then use the fact that $\mathcal{A}_{k}=\mathcal{B}_{k}$. This method is used in the proof of Lemma 12 for the computation of the $\mathcal{S}_{k}$ 's, with $2 \leq k \leq 4$.

Lemma 12. The following identities hold:

$$
\begin{gathered}
\mathcal{S}_{3}=2(\omega+1)\left(\mathcal{T}^{\omega}+2 \omega^{3} \mathcal{M}^{\omega-1}\right), \\
-\mathcal{S}_{2}=2(\omega+1)\left\{\mathcal{T}^{\omega}+4 \omega^{3}\left(\mathcal{T}^{\omega-1}-\mathcal{M}^{\omega-1}\right)+2 \omega^{2}(\omega-1) \mathcal{T}_{1}^{\omega-2}+2 \omega^{2}(\omega-1) \mathcal{B}_{5}\right\}, \\
\mathcal{S}_{4}=4 \omega(\omega+1)\left\{\omega \mathcal{T}^{\omega-1}-\omega(\omega+1) \mathcal{M}^{\omega-1}+(\omega-1) \mathcal{B}_{5}+2 \omega(\omega-1)^{3} \mathcal{N}^{\omega-2}\right\} .
\end{gathered}
$$

Proof. Recall that the $\mathcal{S}_{k}$ 's are defined in the beginning of this section. First, we contract the index $b$, occurring in the symmetric tensor which defines $\mathcal{S}_{2}$, with all the other indices not yet contracted (i.e. the indices in $I \cup J \cup\{a\}$ ), we obtain:

$\frac{-\mathcal{S}_{2}}{2 \omega+2}=\operatorname{Tr} \operatorname{Sym}\left\{\nabla_{I} \operatorname{Ric}_{i j} \nabla_{J} \operatorname{Ric}_{i j}-\omega \nabla_{I^{\prime}} \nabla_{b} R_{i a b j} \nabla_{J} \operatorname{Ric}_{i j}-\omega \nabla_{I} R_{i a b j} \nabla_{J^{\prime}} \nabla_{b} \operatorname{Ric}_{i j}\right\}$,

We continue, by contracting the index $a$ with all possible indices and we obtain:

$$
\begin{aligned}
\frac{-\mathcal{S}_{2}}{2 \omega+2}= & \mathcal{T}^{\omega}-2 \omega^{2}(\omega-1) \operatorname{Tr} \operatorname{Sym}\left\{\nabla_{I^{\prime \prime}} \nabla_{a b} R_{i a b j} \nabla_{J} \operatorname{Ric}_{i j}+\nabla_{I} R_{i a b j} \nabla_{J^{\prime \prime}} \nabla_{a b} \operatorname{Ric}_{i j}\right\}- \\
& 2 \omega^{3} \operatorname{Tr} \operatorname{Sym}\left\{\nabla_{I^{\prime}} \nabla_{b} R_{i a b j} \nabla_{J^{\prime}} \nabla_{a} \operatorname{Ric}_{i j}+\nabla_{I^{\prime}} \nabla_{a} R_{i a b j} \nabla_{J^{\prime}} \nabla_{b} \operatorname{Ric}_{i j}\right\} \\
= & \frac{1}{c_{1}}\left\{c_{1} \mathcal{A}_{1}+c_{2} \mathcal{A}_{2}+c_{3} \mathcal{A}_{3}+c_{2} \mathcal{A}_{4}+c_{3} \mathcal{A}_{5}\right\},
\end{aligned}
$$

where we used the notation of Table 1 . Using the fact that $\mathcal{A}_{k}=\mathcal{B}_{k}$, we obtain the claimed equality for $\mathcal{S}_{2}$. Using the same method, which consists of contracting the entries of the Riemann and Ricci curvature tensors, we compute $\mathcal{S}_{3}$ and $\mathcal{S}_{4}$.

The identity holds for $\mathcal{S}_{3}$, using the contracted second Bianchi identity and the fact that $\mid \nabla^{\omega}$ scal $\mid=0$. For $\mathcal{S}_{4}$, we first contract the index $a$ with all the other not yet contracted indices and obtain:

$$
\begin{aligned}
\frac{\mathcal{S}_{4}}{2 \omega+2}=\operatorname{Tr} \operatorname{Sym}\left\{-\nabla_{I} \operatorname{Ric}_{i j} \nabla_{J^{\prime} i} \operatorname{Ric}_{c j}+\omega \nabla_{I^{\prime} a} R_{i a b j} \nabla_{J^{\prime} i} \operatorname{Ric}_{c j}+\right. \\
\left.(\omega-1) \nabla_{I} R_{i a b j} \nabla_{J^{\prime \prime} i a} \operatorname{Ric}_{c j}+\nabla_{I} R_{i a b j} \nabla_{J^{\prime} i} \operatorname{Ric}_{a j}\right\} .
\end{aligned}
$$

The third term of the right hand side in the last equality vanishes since the Riemann tensor is skew-symmetric with respect to the two first entries and the covariant derivatives of the Ricci tensor commute. By the second Bianchi identity, we have $\nabla_{a} R_{i a b j}=\nabla_{j}$ Ric $_{i b}-\nabla_{b}$ Ric $_{i j}$. Substituting in the second term and using the fact that $\mid \nabla^{\omega}$ scal $\mid=0$, we obtain

$$
\begin{aligned}
\frac{\mathcal{S}_{4}}{2 \omega+2}=\left\{-2 \omega^{2} \mathcal{M}^{\omega-1}+2 \omega^{2}(\omega-1)\left[2(\omega-1)^{2} \mathcal{N}^{\omega-2}-\mathcal{M}^{\omega-1}\right]+\right. \\
\\
\left.2 \omega(\omega-1) \mathcal{A}_{5}+2 \omega^{2} \mathcal{A}_{4}\right\} .
\end{aligned}
$$

Therefore, the equality corresponding to $\mathcal{S}_{4}$ holds, since $\mathcal{A}_{4}=\mathcal{B}_{4}$ and $\mathcal{A}_{5}=\mathcal{B}_{5}$ (cf. Table 1).

End of Theorem 1 proof. By Corollary 7, it is sufficient to prove that

$$
\mathcal{I}:=\operatorname{Tr} \mathcal{Q}(R)+2(\omega+3)\left[(\omega+3)\left(\mathcal{S}_{2}+\mathcal{S}_{3}\right)+\omega \mathcal{S}_{4}\right]>0 .
$$

By Lemma 12, it follows that 


$$
\begin{aligned}
& 2(\omega+3)\left[(\omega+3)\left(\mathcal{S}_{2}+\mathcal{S}_{3}\right)+\omega \mathcal{S}_{4}\right]=4(\omega+3)(\omega+1) \omega^{2}\left\{-2 \omega(2 \omega+5)\left(\mathcal{T}^{\omega-1}-\mathcal{M}^{\omega-1}\right)\right. \\
& \left.-2(\omega-1)(\omega+3) \mathcal{T}_{1}^{\omega-2}-2(\omega-1)(\omega+2) \mathcal{B}_{5}+6 \omega \mathcal{M}^{\omega-1}+4 \omega(\omega-1)^{3} \mathcal{N}^{\omega-2}\right\} .
\end{aligned}
$$

Therefore, by Proposition 10 and (22), we obtain

$$
\begin{gathered}
\frac{\mathcal{I}}{4(\omega+1)}>2 \omega^{3}(\omega+1)\left(\mathcal{T}^{\omega-1}-\mathcal{M}^{\omega-1}\right)+(\omega+2)\left\{\mathcal{T}^{\omega}+8 \omega^{3}(\omega-1)^{2}(\omega-2)\left(\mathcal{T}_{1}^{\omega-3}-\mathcal{M}_{1}^{\omega-3}\right)\right\}+ \\
2 \omega^{2}(\omega-1)\left\{2 \omega(\omega+2)(\omega-1)^{2} \mathcal{T}_{1,1}^{\omega-2}-\left(\omega^{2}+4 \omega+5\right) \mathcal{T}_{1}^{\omega-2}\right\}+ \\
(\omega+3)\left\{6 \omega^{3} \mathcal{M}^{\omega-1}+4 \omega^{3}(\omega-1)^{3} \mathcal{N}^{\omega-2}\right\}
\end{gathered}
$$

where we used the fact that $\mathcal{B}_{5}$ is nonnegative. Set

$$
\begin{aligned}
& \mathcal{I}_{1}:=2 \omega^{2}(\omega-1)\left\{2 \omega(\omega+2)(\omega-1)^{2}\right. \mathcal{T}_{1,1}^{\omega-2}-\left(\omega^{2}+4 \omega+5\right) \mathcal{T}_{1}^{\omega-2}+ \\
&\left.4 \omega(\omega+2)(\omega-1)(\omega-2)\left(\mathcal{T}_{1}^{\omega-3}-\mathcal{M}_{1}^{\omega-3}\right)\right\}, \\
& \mathcal{I}_{2}:=2 \omega^{3}(\omega+1)\left(\mathcal{T}^{\omega-1}-\mathcal{M}^{\omega-1}\right)+(\omega+2) \mathcal{T}^{\omega}+ \\
&(\omega+3)\left\{6 \omega^{3} \mathcal{M}^{\omega-1}+4 \omega^{3}(\omega-1)^{3} \mathcal{N}^{\omega-2}\right\} .
\end{aligned}
$$

In this notation, we have $\mathcal{I}>4(\omega+1)\left(\mathcal{I}_{1}+\mathcal{I}_{2}\right)$. In order to finish the proof, we compute $\mathcal{I}_{1}$ and $\mathcal{I}_{2}$ and show that $\mathcal{I}_{1}+\mathcal{I}_{2} \geq 0$. For the computation of $\mathcal{I}_{2}$, we have

$$
\begin{aligned}
6 \omega^{3} \mathcal{M}^{\omega-1}+4 \omega^{3}(\omega-1)^{3} \mathcal{N}^{\omega-2} & =6 \omega^{3} \sum_{\ell=0}^{\left[\frac{\omega-1}{2}\right]} d_{\ell}^{\omega-1} \mathcal{M}_{\ell}+4 \omega^{3}(\omega-1)^{3} \sum_{\ell=0}^{\left[\frac{\omega-2}{2}\right]} d_{\ell}^{\omega-2} \mathcal{N}_{\ell} \\
& =\sum_{\ell=0}^{\left[\frac{\omega-1}{2}\right]}(\omega-2 \ell) d_{\ell}^{\omega}\left[3 \mathcal{M}_{\ell}+(\omega-2 \ell-1) \mathcal{N}_{\ell}\right]
\end{aligned}
$$

since for $\omega$ odd, $\mathcal{N}_{\frac{\omega-1}{2}}=0$ by definition. We substitute the value of $\mathcal{N}_{\ell}$, given by (5) and obtain

$$
\begin{aligned}
& (\omega+3)\left\{6 \omega^{3} \mathcal{M}^{\omega-1}+4 \omega^{3}(\omega-1)^{3} \mathcal{N}^{\omega-2}\right\}= \\
& \sum_{\ell=0}^{\left[\frac{\omega-1}{2}\right]} d_{\ell}^{\omega}(\omega+3)\left\{-2 \mathcal{T}_{\ell}-(\omega-2 \ell) \mathcal{M}_{\ell}\right\} .
\end{aligned}
$$

If $\omega$ is even, then $\mathcal{T}_{\frac{\omega}{2}}=0$, by (5). Thus

$$
\begin{aligned}
(\omega+2) \mathcal{T}^{\omega} & =\sum_{\ell=0}^{\left[\frac{\omega-1}{2}\right]} d_{\ell}^{\omega}(\omega+2) \mathcal{T}_{\ell} . \\
2 \omega^{3}(\omega+1)\left(\mathcal{T}^{\omega-1}-\mathcal{M}^{\omega-1}\right) & =\sum_{\ell=0}^{\left[\frac{\omega-1}{2}\right]} d_{\ell}^{\omega}(\omega+1)(\omega-2 \ell)\left(\mathcal{T}_{\ell}-\mathcal{M}_{\ell}\right) .
\end{aligned}
$$
by

The sum of the right hand sides of (23), (24) and (25), denoted by $\mathcal{I}_{2}$, is given

$$
\mathcal{I}_{2}=\sum_{\ell=0}^{\left[\frac{\omega-1}{2}\right]} d_{\ell}^{\omega}\left\{[(\omega+1)(\omega-2 \ell)-(\omega+4)] \mathcal{T}_{\ell}-2(\omega-2 \ell)(\omega+2) \mathcal{M}_{\ell}\right\}
$$


Substituting the value of $\mathcal{M}_{\ell}$ given by (5) and using the inequality of Lemma 5, it follows that

$$
\mathcal{I}_{2} \geq \sum_{\ell=0}^{\left[\frac{\omega-1}{2}\right]} 2 \ell d_{\ell}^{\omega} \mathcal{T}_{\ell}
$$

For the computation of $\mathcal{I}_{1}$, we proceed similarly as above. By equalities (16) and (17) we have

$$
\begin{aligned}
-2 \omega^{2}(\omega-1)\left(\omega^{2}+4 \omega+5\right) \mathcal{T}_{1}^{\omega-2} & =-\sum_{\ell=1}^{\left[\frac{\omega-1}{2}\right]} 2 \ell\left(\omega^{2}+4 \omega+5\right) d_{\ell}^{\omega} \mathcal{T}_{\ell}, \\
4 \omega^{3}(\omega+2)(\omega-1)^{3} \mathcal{T}_{1,1}^{\omega-2} & =\sum_{\ell=1}^{\left[\frac{\omega-1}{2}\right]} 4 \ell^{2}(\omega+2) d_{\ell}^{\omega} \mathcal{T}_{\ell}, \\
8 \omega^{3}\left(\omega^{2}-4\right)(\omega-1)^{2}\left(\mathcal{T}_{1}^{\omega-3}-\mathcal{M}_{1}^{\omega-3}\right) & =\sum_{\ell=1}^{\left[\frac{\omega-1}{2}\right]} 4 \ell(\omega+2)(\omega-2 \ell) d_{\ell}^{\omega}\left(\mathcal{T}_{\ell}-\mathcal{M}_{\ell}\right) .
\end{aligned}
$$

Taking the sum of the last three equalities, we obtain

$$
\mathcal{I}_{1}=\sum_{\ell=1}^{\left[\frac{\omega-1}{2}\right]} 2 \ell d_{\ell}^{\omega}\left\{[\omega(\omega-2 \ell)-4 \ell-5] \mathcal{T}_{\ell}-2(\omega-2 \ell)(\omega+2) \mathcal{M}_{\ell}\right\} .
$$

Hence

$$
\mathcal{I}_{1} \geq-\sum_{\ell=1}^{\left[\frac{\omega-1}{2}\right]} 2 \ell d_{\ell}^{\omega} \mathcal{T}_{\ell}
$$

By (26) and (27), we conclude that $\mathcal{I}>4(\omega+1)\left(\mathcal{I}_{1}+\mathcal{I}_{2}\right) \geq 0$.

\section{Appendix A.}

A.1. Proof of the equalities $\mathcal{A}_{k}=\mathcal{B}_{k}$. In the following computation, the components of the Ricci tensor Ric are denoted by $R_{i j}$. Here we give some useful formulas for the computation of the $\mathcal{A}_{k}$ 's:

$$
\begin{gathered}
\nabla_{i} R_{i j}=\frac{1}{2} \nabla_{j} \text { scal } \\
\nabla_{a} R_{i a b j}=-\nabla_{b} R_{i j}+\nabla_{j} R_{b i} \text { (by } 2^{\text {nd }} \text { Bianchi) } \\
\nabla_{b} R_{i a b j}=-\nabla_{a} R_{i j}+\nabla_{i} R_{a j} \\
\Delta R_{i a b j}=\nabla_{b i} R_{a j}+\nabla_{a j} R_{b i}-\nabla_{i j} R_{a b}-\nabla_{a b} R_{i j} . \quad\left(\text { by } 2^{\text {nd }} \text { Bianchi }\right) \\
\mathcal{A}:=\nabla_{c} R_{i a b j} \cdot \nabla_{c} R_{i b a j}=-\nabla_{c} R_{i a b j} \cdot\left(\nabla_{c} R_{b a i j}+\nabla_{c} R_{a i b j}\right) \\
=\mid \nabla \text { Riem }\left.\right|^{2}-\mathcal{A}, \quad \text { yielding } \mathcal{A}=\frac{1}{2} \mid \nabla \text { Riem }\left.\right|^{2} . \\
\mathcal{B}:=\nabla_{c} R_{i a b j} \cdot \nabla_{b} R_{i a c j}=-\nabla_{c} R_{i a b j} \cdot\left(\nabla_{j} R_{i a b c}+\nabla_{c} R_{i a j b}\right) \\
=\mid \nabla \text { Riem }\left.\right|^{2}-\mathcal{B}, \quad \text { yielding } \mathcal{B}=\frac{1}{2} \mid \nabla \text { Riem }\left.\right|^{2} .
\end{gathered}
$$

$\mathcal{A}_{1}:=\mathcal{T}^{\omega}$.

$\mathcal{A}_{2}:=-\operatorname{Tr} \operatorname{Sym} \nabla_{I^{\prime} c} R_{i j} \nabla_{J^{\prime} b} R_{i b c j} \stackrel{(\mathrm{A}-2)}{=} \operatorname{Tr} \operatorname{Sym} \nabla_{I^{\prime} c} R_{i j} \nabla_{J^{\prime}}\left(\nabla_{c} R_{i j}-\nabla_{j} R_{c i}\right)=\mathcal{T}^{\omega-1}-\mathcal{M}^{\omega-1}$.

$\mathcal{A}_{3}:=-\operatorname{Tr} \operatorname{Sym} \nabla_{I} R_{i j} \nabla_{J^{\prime \prime} b c} R_{i b c j} \stackrel{(\mathrm{A}-3)}{=}-\operatorname{Tr} \operatorname{Sym} \nabla_{I} R_{i j} \nabla_{J^{\prime \prime} b}\left(-\nabla_{b} R_{i j}+\nabla_{i} R_{b j}\right)=\mathcal{T}_{1}^{\omega-2}$.

$\mathcal{A}_{4}=-\operatorname{Tr} \operatorname{Sym} \nabla_{I^{\prime} b} R_{i j} \nabla_{J^{\prime} c} R_{i b c j} \stackrel{(\mathrm{A}-3)}{=} \operatorname{Tr} \operatorname{Sym} \nabla_{I^{\prime} b} R_{i j} \nabla_{J^{\prime}}\left(\nabla_{b} R_{i j}-\nabla_{i} R_{b j}\right)=\mathcal{A}_{2}$. $\mathcal{A}_{6}:=\mathcal{R}^{\omega}$. 


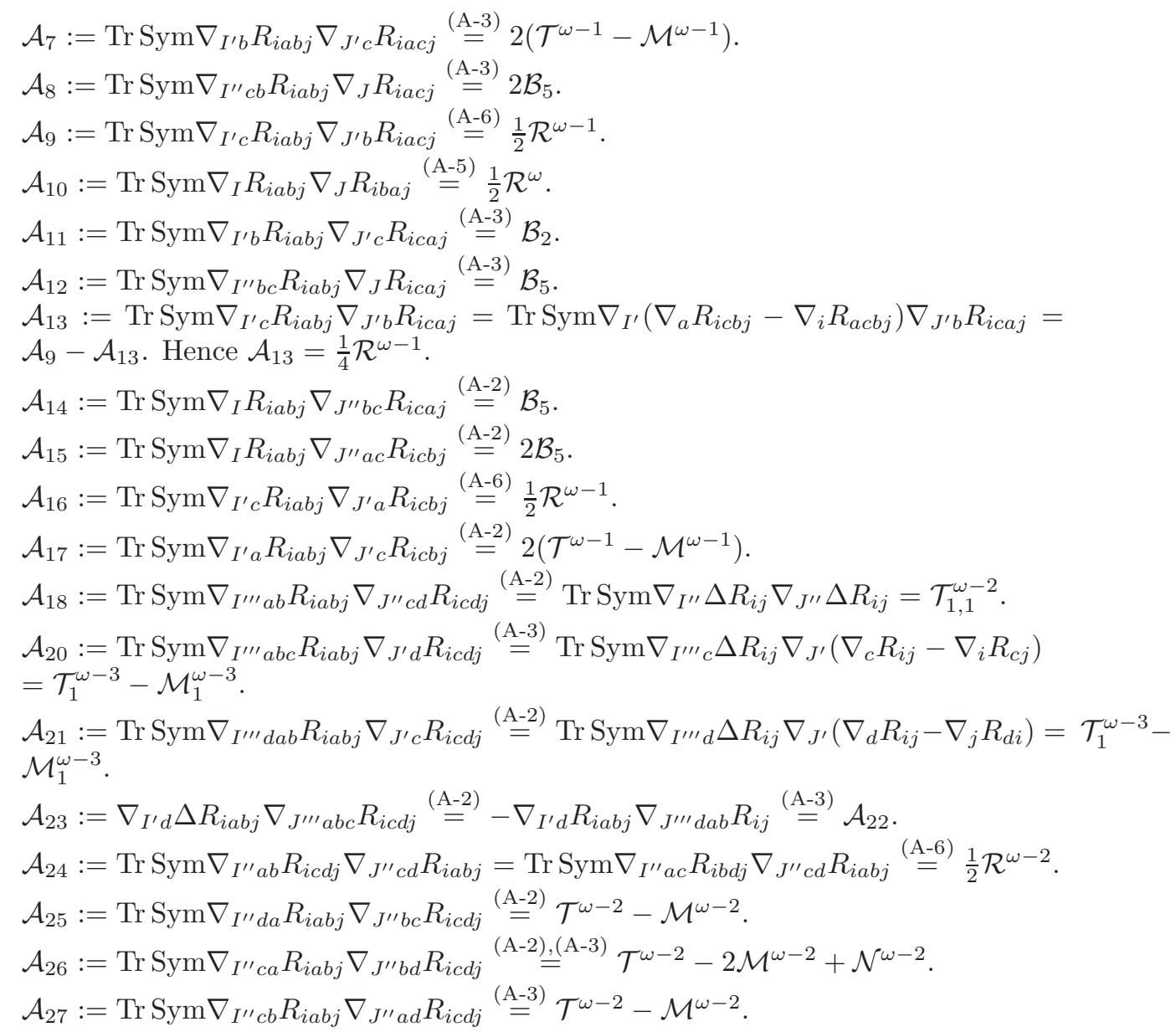

For the remaining terms $\mathcal{A}_{5}, \mathcal{A}_{19}$ and $\mathcal{A}_{22}$, the computation is done by induction, by introducing the following sequence $\mathcal{U}_{\omega-\beta}:=-\operatorname{Tr} \operatorname{Sym} \nabla_{I_{\beta} b c} \nabla_{K_{\beta}} \operatorname{Ric}_{i j} \nabla_{J_{\beta}} \nabla_{K_{\beta}} R_{i b c j}$ for $1 \leq \beta \leq \omega-2$ and $\mathcal{U}_{\omega}:=\mathcal{A}_{5}$, where $K_{\beta}, I_{\beta}$ and $J_{\beta}$ are multi-indices sets of cardinalities $\beta, \omega-\beta-2$ and $\omega-\beta$ respectively. The induction formula is given by

$$
\begin{aligned}
& \mathcal{U}_{\omega-\beta}=2(\omega-\beta-1)(\omega-\beta-2) \mathcal{U}_{\omega-\beta-1}- \\
& 2(\omega-\beta-1)^{2} \operatorname{Tr} \operatorname{Sym} \nabla_{I_{\beta} b c} \nabla_{K_{\beta}} \operatorname{Ric}_{i j} \nabla_{J_{\beta-2}} \nabla_{K_{\beta}} \Delta R_{i b c j} .
\end{aligned}
$$

Using (A-4), we have $\operatorname{Tr} \operatorname{Sym} \nabla_{I_{\beta} b c} \nabla_{K_{\beta}} \operatorname{Ric}_{i j} \nabla_{J_{\beta-2}} \nabla_{K_{\beta}} \Delta R_{i b c j}=\mathcal{T}^{\omega-\beta-2}-2 \mathcal{M}^{\omega-\beta-2}+$ $\mathcal{N}^{\omega-\beta-2}$. By induction on $\beta$, we prove that

$$
\begin{gathered}
\mathcal{A}_{5}=\mathcal{U}_{\omega}=(\omega-1) !(\omega-2) ! \sum_{k=0}^{\omega-2} 2^{\omega-k-1} \frac{k+1}{(k !)^{2}}\left(\mathcal{T}^{k}-2 \mathcal{M}^{k}+\mathcal{N}^{k}\right) \\
\stackrel{[16),(\mathrm{A}-10)}{=} \sum_{\ell=0}^{\left.\frac{\omega-2}{2}\right]} e_{\ell}^{\omega}\left(\mathcal{T}_{\ell}-2 \mathcal{M}_{\ell}+\mathcal{N}_{\ell}\right) .
\end{gathered}
$$

We have $\mathcal{A}_{22}:=\operatorname{Tr} \operatorname{Sym} \nabla_{I^{\prime} c} R_{i a b j} \nabla_{J^{\prime \prime \prime} a b d} R_{i c d j} \stackrel{(\mathrm{A}-3)}{=}-\operatorname{Tr} \operatorname{Sym} \nabla_{I^{\prime} c} R_{i a b j} \nabla_{J^{\prime \prime \prime} a b c} R_{i j}$. By contracting an index in $\mathcal{A}_{5}$, which corresponds to a covariant derivative of the Riemann tensor and using the last equality, we obtain $\mathcal{A}_{5}=2(\omega-1)^{2} \mathcal{B}_{26}+2(\omega-$ 1) $(\omega-2) \mathcal{A}_{22}$. Therefore, by $(\mathrm{A}-7)$, we obtain $\mathcal{A}_{22}=\sum_{\ell=0}^{\left[\frac{\omega-3}{2}\right]} e_{\ell}^{\omega-1}\left(\mathcal{T}_{\ell}-2 \mathcal{M}_{\ell}+\mathcal{N}_{\ell}\right)$. 
We have $\mathcal{A}_{19}:=\operatorname{Tr} \operatorname{Sym} \nabla_{I^{\prime \prime \prime \prime} a b c d} R_{i a b j} \nabla_{J} R_{i c d j}=-\operatorname{Tr} \operatorname{Sym} \nabla_{I^{\prime \prime \prime \prime} c d} \Delta R_{i j} \nabla_{J} R_{i c d j}$. By contracting an index in $\mathcal{A}_{5}$, which corresponds to a covariant derivative of the Ricci tensor and using the last equality, we obtain $\mathcal{A}_{5}=2(\omega-1)(\omega-3) \mathcal{A}_{19}+$ $2 \omega(\omega-1) \mathcal{A}_{22}$. We deduce that

$$
\mathcal{A}_{19}=\sum_{\ell=0}^{\left[\frac{\omega-2}{2}\right]} \frac{\ell e_{\ell}^{\omega}}{(\omega-1)(\omega-2)(\omega-3)}\left(\mathcal{T}_{\ell}-2 \mathcal{M}_{\ell}+\mathcal{N}_{\ell}\right) .
$$

A.2. Combinatorics Formulas. The following identities hold for any nonnegative integer $\omega$

$$
\begin{gathered}
(\omega+2) \sum_{k=\omega}^{2 \omega}(k+1)\left(\begin{array}{l}
k \\
\omega
\end{array}\right)=(\omega+3)^{2} C(\omega), \\
(\omega+2) \sum_{k=\omega}^{2 \omega-1}(k+1)(2 \omega-k)\left(\begin{array}{l}
k \\
\omega
\end{array}\right)=\omega(\omega+3) C(\omega),
\end{gathered}
$$

where $C(\omega)=(\omega+1)^{2}(\omega+2)^{2}(2 \omega+2) ![(\omega+3) !]^{-2}$.

Proof. Identities (A-8) and (A-9) can be written in a simpler way which can be viewed as special cases (for $n=2 \omega$ ) of the following combinatorial identities:

$$
\sum_{k=\omega}^{n-1}\left(\begin{array}{l}
k \\
\omega
\end{array}\right)=\left(\begin{array}{c}
n \\
\omega+1
\end{array}\right), \quad \sum_{k=\omega}^{n-2}(n-k-1)\left(\begin{array}{l}
k \\
\omega
\end{array}\right)=\left(\begin{array}{c}
n \\
\omega+2
\end{array}\right),
$$

for all integers $n \geq \omega$, respectively $n \geq \omega+2$.

These identities follow by counting in a different way the number of combinations. The first identity is obtained by counting the number of subsets with $(\omega+1)$ elements out of $n$ elements in the following way: the sets are separated with respect to their largest element. For each $\omega \leq k \leq n-1,\left(\begin{array}{c}k \\ \omega\end{array}\right)$ counts the subsets of $(\omega+1)$ elements whose largest element is $k+1$.

Similarly, the second identity follows by counting $\left(\begin{array}{c}n \\ \omega+2\end{array}\right)$ as follows: the sets are separated with respect to their second largest element. For each $\omega \leq k \leq n-2$, $(n-k-1)\left(\begin{array}{l}k \\ \omega\end{array}\right)$ is the number of subsets with $\omega+2$ elements whose second largest element is $k+1$. Indeed, if the second largest element is $k+1$, the others $\omega$ elements of the set which are smaller must form a subset of $\omega+k$ and the largest element may be any of the remaining $n-k-1$ elements. 


\section{Notation INDEX}

A

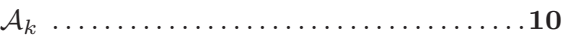

B

$\mathcal{B}_{h}$

$C(\omega)=\frac{(\omega+1)^{2}(\omega+2)^{2}(2(\omega+1)) !}{[(\omega+3) !]^{2}} \ldots \ldots \ldots 3$

$c_{1}, \ldots, c_{6} \ldots \ldots \ldots \ldots \ldots \ldots \ldots \ldots \ldots \ldots$

D

$d_{\ell}^{\gamma}=\frac{2^{\gamma-2 \ell}(\gamma !)^{3}}{(\gamma-2 \ell) !(\ell !)^{2}}$

E

$e_{\ell}^{\gamma}=\frac{2^{\gamma-2 \ell-2} \gamma !(\gamma-1) !(\gamma-2) !}{(\gamma-2 \ell-2) !(\ell+1) ! \ell !}$

$\mathcal{I}$

$\mathcal{I}_{1}, \mathcal{I}_{2} \ldots \ldots \ldots \ldots \ldots \ldots \ldots \ldots \ldots$

$I, J$ multi-indices sets $\# I=\# J=\omega \ldots 10$

$I^{\prime}, I^{\prime \prime}$ multi-indices sets

$\# I^{\prime}+1=\# I^{\prime \prime}+2=\omega$

M

$\mathcal{M}_{\ell}=\nabla_{K} \nabla_{a} \Delta^{\ell} \operatorname{Ric}_{b c} \cdot \nabla_{K} \nabla_{c} \Delta^{\ell} \operatorname{Ric}_{a b} \ldots 3$

$\mathcal{M}_{\ell}^{\omega-1-\beta-2 \ell}=$

$\operatorname{Tr} \underset{I^{\prime} \cup J^{\prime}}{\operatorname{Sym}} \nabla_{I^{\prime} K p} \Delta^{\ell} \operatorname{Ric}_{i j} \nabla_{J^{\prime} K i} \operatorname{Ric}_{p j} \quad \ldots 8$

$\mathbf{N}$

$\mathcal{N}_{\ell}=\nabla_{K^{\prime}} \nabla_{c d} \Delta^{\ell} \operatorname{Ric}_{a b} \nabla_{K^{\prime}} \nabla_{a b} \Delta^{\ell} \operatorname{Ric}_{c d} \ldots \mathbf{3}$

$\mathcal{N}_{\ell}^{\omega-2-\beta-2 \ell}=$

$\operatorname{Tr} \underset{\operatorname{Sym}^{\prime \prime} \cup J^{\prime \prime}}{ } \nabla_{I^{\prime \prime} K p q} \Delta^{\ell} \operatorname{Ric}_{i j} \nabla_{J^{\prime \prime} K i j} \operatorname{Ric}_{p q}$

8

$\mathrm{O}$

$\omega=\inf \left\{k \in \mathbb{N}:\left|\nabla^{k} W e y l_{g}(x)\right| \neq 0\right\} \ldots .2$

a

$\mathcal{Q}(R)_{I J a b c d}=\nabla_{I} R_{i a b j} \nabla_{J} R_{i c d j} \ldots \ldots \ldots \mathbf{5}$

$\mathbf{R}$

$\mathcal{R}_{\ell}=\mid \nabla^{\omega-2 \ell} \Delta^{\ell}$ Riem $\left.\right|^{2} \ldots \ldots \ldots \ldots \ldots \ldots .3$

$\mid \nabla^{k}$ Riem $\mid k$-th derivative norm of Riem . 2 $\operatorname{Ric}_{i j}=R_{i \ell j}^{\ell} \ldots \ldots \ldots \ldots \ldots \ldots \ldots \ldots \ldots$

$R_{j k \ell}^{q} X^{j}=\nabla_{k} \nabla_{\ell} X^{q}-\nabla_{\ell} \nabla_{k} X^{q} \ldots \ldots \ldots 1$

$R_{i j k \ell}=g_{i q} R_{j k \ell}^{q} \ldots \ldots \ldots \ldots \ldots \ldots \ldots 1$

$\mathbf{S}$

$\mathfrak{S}(k)$ symmetric group of $\{1, \ldots, k\} \ldots .2$

$\mathcal{S}=\operatorname{Tr} \quad$ Sym $\quad \nabla_{K} \mathrm{Ric}_{a b} \ldots \ldots 5$

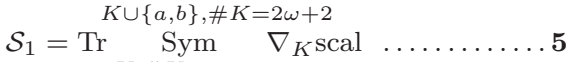

$\mathcal{S}_{2}=\operatorname{Tr} \stackrel{K, \# K=2 \omega+2}{\operatorname{Sym}} \nabla_{I} \operatorname{Ric}_{a j} \cdot \nabla_{J} \mathrm{Ric}_{b j} \ldots 5$

$\mathcal{S}_{2}=\operatorname{Tr} \underset{I \cup J \cup\{a, b\},}{\operatorname{Sym}}, \nabla_{I} \operatorname{Ric}_{a j} \cdot \nabla_{J} \operatorname{Ric}_{b j} \ldots .5$

$\mathcal{S}_{3}=\operatorname{Tr} \underset{\operatorname{Sym}}{\operatorname{Sym}} \nabla_{I} \operatorname{Ric}_{a j} \cdot \nabla_{J} \mathrm{Ric}_{b j} \ldots \mathbf{5}$ $I \cup J \cup\{a, b\}$,
$\# I=\# J=\omega$

$\mathcal{S}_{4}=\operatorname{Tr}{ }^{\mathrm{Sym}} \nabla_{I} R_{i a b j} \cdot \nabla_{J^{\prime}} \nabla_{i} \mathrm{Ric}_{c j} \mathbf{5}$ $I \cup J^{\prime} \cup\{a, b, c\}$,
$\# I=\# J^{\prime}+1=\omega$

$\operatorname{Sym} T_{p_{1} \ldots p_{k}}=\sum_{\sigma \in \mathfrak{S}(k)} T_{p_{\sigma(1)} \ldots p_{\sigma(k)}} \ldots \ldots 2$
$\mathbf{T}$

$\mathcal{T}_{\ell}=\mid \nabla^{\omega-2 \ell} \Delta^{\ell}$ Ric $\left.\right|^{2} \ldots \ldots \ldots \ldots \ldots \ldots .3$

$\mathcal{T}_{\ell}^{\omega-\beta-2 \ell}=\operatorname{Tr} \operatorname{Sym}_{I K} \nabla^{\ell} \operatorname{Ric}_{i j} \nabla_{J K} \operatorname{Ric}_{i j}$

$\mathcal{T}_{1,1}^{\omega-2}=\operatorname{Tr} \operatorname{Sym}_{I^{\prime \prime} \cup J^{\prime \prime}} \nabla_{I^{\prime \prime}} \Delta \operatorname{Ric}_{i j} \nabla_{J^{\prime \prime}} \Delta \operatorname{Ric}_{i j} \quad \mathbf{8}$

$\mathcal{T}^{\omega-\beta}=\mathcal{T}_{0}^{\omega-\beta} \ldots \ldots \ldots \ldots \ldots \ldots \ldots \ldots$.

$\mathcal{T}_{\ell}^{0}=(2 \ell) ! \mathcal{T}_{\ell} \ldots \ldots \ldots \ldots \ldots \ldots \ldots .8$

$\operatorname{Tr} \operatorname{Sym} T=g^{p_{1} p_{2}} \ldots g^{p_{k-1} p_{k}} \operatorname{Sym} T_{p_{1} \ldots p_{k}} \quad \mathbf{2}$ 


\section{REFERENCES}

1. T. Aubin, Sur quelques problèmes de courbure scalaire, J. Funct. Anal 240 (2006), 269-289.

2. __ Solution complète de la $C^{0}$ compacité de l'ensemble des solutions de l'équation de Yamabe, J. Funct. Anal. 244 (2007), 579-589.

3. - On the $C^{0}$ compactness of the set of the solutions of the Yamabe equation, Bull. Sci. Math. 133 (2009), no. 2, 186-189.

4. S. Brendle, Blow-up phenomena for the Yamabe equation., J. Amer. Math. Soc. 21 (2008), no. 4, 951-979.

5. S. Brendle and F. C. Marques, Blow-up phenomena for the Yamabe equation. II, J. Diff. Geom. 81 (2009), no. 2, 225-250.

6. E. Hebey and M. Vaugon, Le problème de Yamabe équivariant, Bull. Sci. Math. 117 (1993), 241-286.

7. J.M. Lee and T. Parker, The Yamabe problem, Bull. Amer. Math. Soc. 17 (1987), 37-91.

8. F. Madani, Equivariant Yamabe problem and Hebey-Vaugon conjecture, J. Func. Anal. 258 (2010), 241-254.

9. _ Hebey-Vaugon conjecture II, C. R. Acad. Sci. Paris Ser I 350 (2012), 849-852.

Fakultät für Mathematik, Universität Regensburg, 93053 Regensburg, Germany

E-mail address: Farid.Madani@mathematik.uni-regensburg.de 